\title{
Article \\ Open-Source Coprocessor for Integer Multiple Precision Arithmetic
}

\author{
Kamil Rudnicki ${ }^{1}$, Tomasz P. Stefański ${ }^{2, *(1)}$ and Wojciech Żebrowski ${ }^{3}$ \\ 1 Department of Reconfigurable Systems, Brightelligence Inc., Glasgow G20 6LL, UK; \\ kamil.rudnicki@brightelligence.co.uk \\ 2 Faculty of Electronics, Telecommunications and Informatics, Gdansk University of Technology, \\ 80-233 Gdansk, Poland \\ 3 Aldec Inc., 80-288 Gdansk, Poland; wojtekz@aldec.com \\ * Correspondence: tomasz.stefanski@pg.edu.pl; Tel.: +48-58-348-63-23
}

Received: 27 May 2020; Accepted: 11 July 2020; Published: 14 July 2020

check for updates

\begin{abstract}
This paper presents an open-source digital circuit of the coprocessor for an integer multiple-precision arithmetic (MPA). The purpose of this coprocessor is to support a central processing unit (CPU) by offloading computations requiring integer precision higher than 32/64 bits. The coprocessor is developed using the very high speed integrated circuit hardware description language (VHDL) as an intellectual property (IP) core. Therefore, it can be implemented within field programmable gate arrays (FPGAs) at various scales, e.g., within a system on chip (SoC), combining CPU cores and FPGA within a single chip as well as FPGA acceleration cards. The coprocessor handles integer numbers with precisions in the range 64 bits-32 kbits, with the limb size set to 64 bits. In our solution, the sign-magnitude representation is used to increase the efficiency of the multiplication operation as well as to provide compatibility with existing software libraries for MPA. The coprocessor is benchmarked in factorial $(n !)$, exponentiation $\left(n^{n}\right)$ and discrete Green's function (DGF) computations on Xilinx Zynq-7000 SoC on TySOM-1 board from Aldec. In all benchmarks, the coprocessor demonstrates better runtimes than a CPU core (ARM Cortex A9) executing the same computations using a software MPA library. For sufficiently large input parameters, our coprocessor is up to three times faster when implemented in FPGA on SoC, rising to a factor of ten in DGF computations. The open-source coprocessor code is licensed under the Mozilla Public License.
\end{abstract}

Keywords: FPGAs; multiple-precision arithmetic; scientific computing; coprocessors

\section{Introduction}

It is a recurrent theme that the current state of computing technology is inadequate to simulate topical scientific problems. Although computer technology is progressing very fast, there are still problems that cannot be solved using the standard numerical precision. Our present work is motivated by the growth in importance of scientific and engineering computations in multiple-precision arithmetic (MPA), i.e., precision higher than the standard 32/64 bits.

In general, MPA delivers methods to solve numerically difficult problems. It is predicted in the scientific literature that [1] "we may be witnessing the birth of a new era of scientific computing, in which the numerical precision required for a computation is as important to the program design as are the algorithms and data structures". Therefore, MPA has already found applications in scientific computing, e.g., it has been used in computational electromagnetics for many years not only by us [2-5] but also by other researchers [6-8].

According to Ref. [1], MPA is useful for: 
- Solving ill-conditioned linear systems of equations; many scientific problems involve ill-conditioned linear systems that give rise to numerical errors even when using 64-bit floating-point arithmetic.

- Computing large sums; anomalous results often stem from the loss of associativity in summations, wrong order of summations or the usage of inadequate algorithms $[9,10]$.

- Executing long-time simulations; almost any kind of physical simulation (e.g., N-body simulations [11,12]) is sensitive to cumulative round-off errors, in addition to errors arising from discretization of time and space.

- Executing large-scale simulations; computations that are well-behaved on modest-size problems may exhibit significant numerical errors when scaled up to massively parallel systems, because making numerical computations parallel is not trivial [12].

- Resolving small-scale phenomena; very fine-scale resolutions can result in numerical problems.

- "Experimental mathematics" computations; numerous recent results in experimental mathematics could not be obtained without MPA computations.

In our opinion, it is necessary also to add to this list other important MPA applications such as:

- Generation of special mathematical functions applicable in scientific computations (e.g., discrete Green's function (DGF), Bessel functions, etc.).

- Cryptography and cryptanalysis.

Unfortunatelly, MPA applications involve large computing resources, i.e., processor time and memory consumption. According to [1], computations on central processing units (CPUs) in double-double precision typically run 5-10 times slower than those implemented in 64-bit arithmetic. The slowdown is at least 25 times for quad-double arithmetic, more than 100 times for 100-digit arithmetic, and over 1000 times for 1000-digit arithmetic. This means that the development of a parallel MPA accelerator is currently vitally important for scientists who need to apply MPA in their computations.

Ref. [13] presents an arithmetic unit for variable high-precision operands suitable for reconfigurable computing. This unit cooperates with a host computer. While this is regarded as a seminal solution, it only accelerates arithmetic operations (i.e., multiplication, division and square root) and does not allow for execution of dedicated codes. In [14], hardware designs, arithmetic algorithms, and software support are presented for a family of variable-precision, interval arithmetic processors. The aim of such processors is to provide a programmer the ability to specify the precision of computations, determine the accuracy of the results and, if desired, correct implicit errors in finite-precision computations. Unfortunately, these processor designs are only specified and simulated at the behavioural level, and no physical implementation has been made. In [15], a special-purpose very large instruction word processor for variable-precision arithmetic is presented, which uses unified hardware to implement various algebraic and transcendental functions. Its performance is obtained by using the explicitly parallel nature of the very large instruction word and dynamically varying the precision of intermediate computations. A prototype of that processor, as well as an accelerator, are implemented in a field-programmable gate array (FPGA), which provides 5-37 times of speedup compared with a software library. In [16,17], the development of a series of boards is reported, which accelerate scientific computations requiring high precision. These boards consist of processing elements, including a floating-point multiply unit and an addition unit, which form a single-instruction multiple-data processor. Although these hardware solutions are 80-200 times faster than corresponding software implementations, their computations are limited to quadruple, hexuple, and octuple precision arithmetic. In [18-20], a variable-precision floating-point accelerator is proposed, based on a refined version of the universal number (UNUM) type I format, for high-performance-computing servers. It is implemented as a coprocessor of the RISC-V processor generated with the Rocket-chip generator [21]. This design is prototyped and validated on FPGA. In general, the FLOPS performance of this 
architecture stays within the range of a regular fixed-precision IEEE floating-point unit. Furthermore, the UNUM format allows interval arithmetic to be used in real applications having a variable memory footprint.

Up to now, none of the MPA processor/coprocessor results [13-20] presented in the literature have gained either immense popularity or worldwide success. In our opinion, it stems partially from the fact that none of those solutions are freely available as an open-source intellectual property (IP) core. As a result, none of these solutions are available as a parallel computing coprocessor for MPA. Despite the passage of time, the design of MPA processors/coprocessors remains an open scientific problem, which we have also tried to solve [22-24]. Now, an MPA coprocessor is developed with scalability, enabling its implementation in FPGAs of various scale using a single IP core. In this work, the coprocessor is implemented on system on chip (SoC) devices (i.e., Zynq devices from Xilinx [25]), but the design can also be implemented in FPGA PCIe accelerator cards. To the best of the Authors' knowledge, this is the only recent integer MPA coprocessor which is both presented in the literature and whose source code is available to the public.

Recently, preliminary results have been reported from implementations of various MPA operations [22,24] as well as the concept of the IP core for the integer MPA coprocessor [23]. In this paper, a comprehensive and expanded report on this research is presented, delivering a significant extension on the previous results. The new results in this paper focus on the acceleration of factorial ( $n !)$, exponentiation $\left(n^{n}\right)$ and DGF computations on Xilinx Zynq-7000 SoC. In all benchmarks, the integer MPA coprocessor demonstrates advantages in terms of runtime in comparison with a CPU core executing the same computations using a software MPA library. For sufficiently large input parameters, our integer MPA coprocessor (implemented in FPGA on Zynq-7000 SoC) is up to three times faster than a single CPU core within the same chip (ARM Cortex A9) emulating MPA using a software library. It is important to state that the code of our coprocessor is open sourced and licensed under the Mozilla Public License [26]. It can hopefully facilitate the investigations of other researchers who employ MPA, because not only has the IP core of the MPA coprocessor been released but also the emulator and benchmarking codes presented here.

\section{Architecture of Coprocessor}

The very high speed integrated circuit hardware description language (VHDL) code of the MPA coprocessor is developed for implementation in Xilinx Zynq-7000 SoC on TySOM-1 board from Aldec [27] consisting of Kintex-7 architecture FPGA and two 32-bit ARM Cortex A9 CPU cores (package XC7Z030-1FBG484C). The architecture of Zynq-7000 SoC with the MPA coprocessor is presented in Figure 1. The processing system (PS) communicates with the programmable logic (PL) using an AXI interface [28], requiring AXI interconnect and dedicated converters. In order to resolve the problem of data integrity, the first-in first-out (FIFO) buffers are used for data storage and buffering between PS and the MPA coprocessor. For the purpose of the coprocessor benchmarking in Section 4, the block random access memory (BRAM) is connected to the MPA coprocessor through the AXI interface. However, it can be removed in the target coprocessor configuration.

Our aim is to develop an MPA coprocessor core that can be implemented in various FPGAs, according to the required processing power of the final application. The IP core is parametrizable and uses the sign-magnitude representation for integer numbers. This stems from the need to provide an interface compatible with existing software codes and libraries employing MPA, such as the GMP library [29], which is the standard software tool for implementation of MPA computations on CPUs. Whilst the sign-magnitude representation of integer numbers is not so straightforward for addition/subtraction implementation (requiring $O(n)$ operations) as two's complement representation, it delivers time savings for the multiplication which requires $O\left(n^{2}\right)$ operations. The format of integer numbers in our MPA coprocessor is presented in Figure 2. In our design, each number consists of a header (64 bits) and data words (limbs) of 64 bits each. The header includes 9 bits of size (i.e., number of limbs), 1 bit of a number sign and 3 bits of control flags for speeding-up processing on FPGA. 


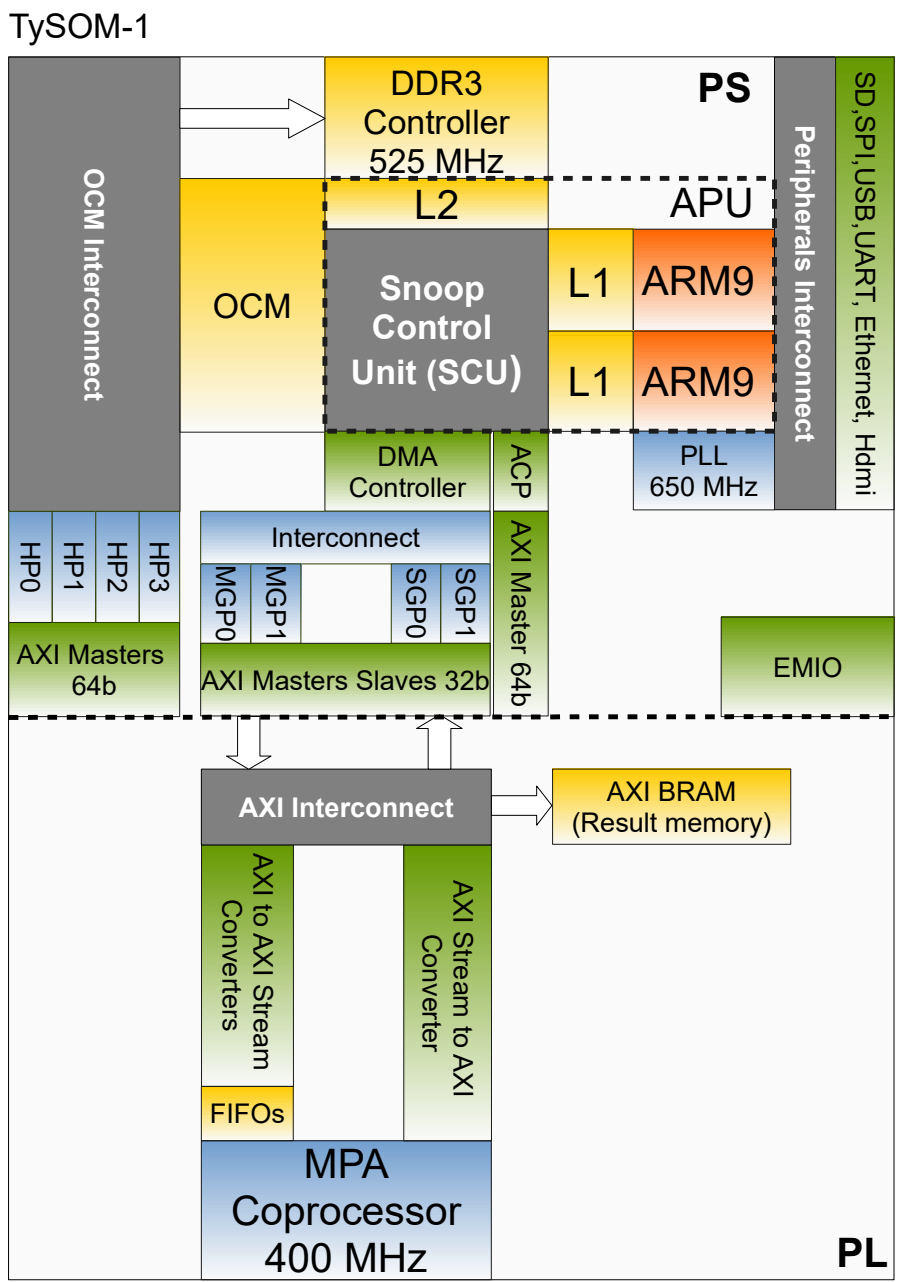

Figure 1. Architecture of Zynq-7000 SoC with implemented multiple-precision arithmetic (MPA) coprocessor.

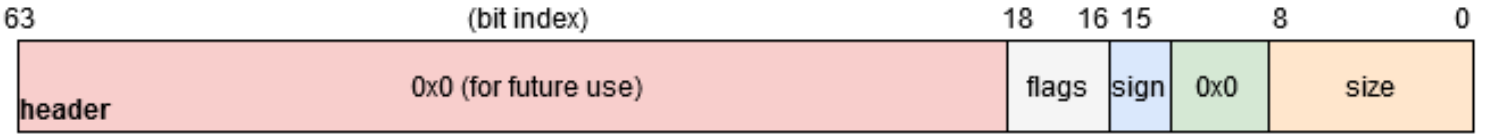

64 data bits $\quad \operatorname{limb} 0$ (least significant limb)

64 data bits $\quad \operatorname{limb} 1$

\section{4 data bits \\ Figure 2. Format of integer numbers in MPA coprocessor.}

limb (size-1) (most significant limb)

The overall diagram of our coprocessor is presented in Figure 3. In the basic configuration, the coprocessor operates under control of host CPU. It exchanges data with host CPU using three 64-bit data buses and a single 8-bit program bus. Two of the data buses (denoted as A and B) transfer data to the coprocessor whilst the third-one transfers results of computations from the MPA coprocessor to CPU. The buses are AMBA standard (AXI Stream) compliant [28]. There are 16 logical registers that can be used in coprocessor codes. From the hardware point of view, those registers are mapped into 17 physical registers. One extra physical register is utilized for operations that may not produce an 
immediately unambiguous result. Extra result data is stored in it (e.g., during addition/subtraction operation) and the proper result selection is made once the operation terminates. This additional register is referred to as the shadow register. Whereas registers store magnitudes of MPA numbers, their signs and sizes are stored in the control unit (CTRL). Loaders transfer data to the bank of registers from data buses A and B whereas unloaders transfer data to CPU. Each register in the bank stores an MPA number of the maximal length equal to $32 \mathrm{kbits}$. When instructions are fetched from the program bus, they are decoded in the instruction decoder, then the control lines (Ctrl) are set accordingly to allow data transfer from the registers to the multiplication and adder/subtractor units. Then, arithmetic operations are executed and the results are transferred back to the bank of registers. The final results of the MPA computations are transferred to CPU using the unloader module.

The control unit is responsible for fetching instructions for execution, instruction decoding, and mapping register numbers from the logical representation given by the user to physical representation in hardware. It executes instructions provided that all necessary resources are available, otherwise it awaits them. When all resources required by the current operation are ready, the CTRL unit generates appropriate control signals on the control lines. The control unit also monitors the amount of individual resources, temporarily blocks or allows the next instruction to be executed, and stores additional information supporting fast number processing.
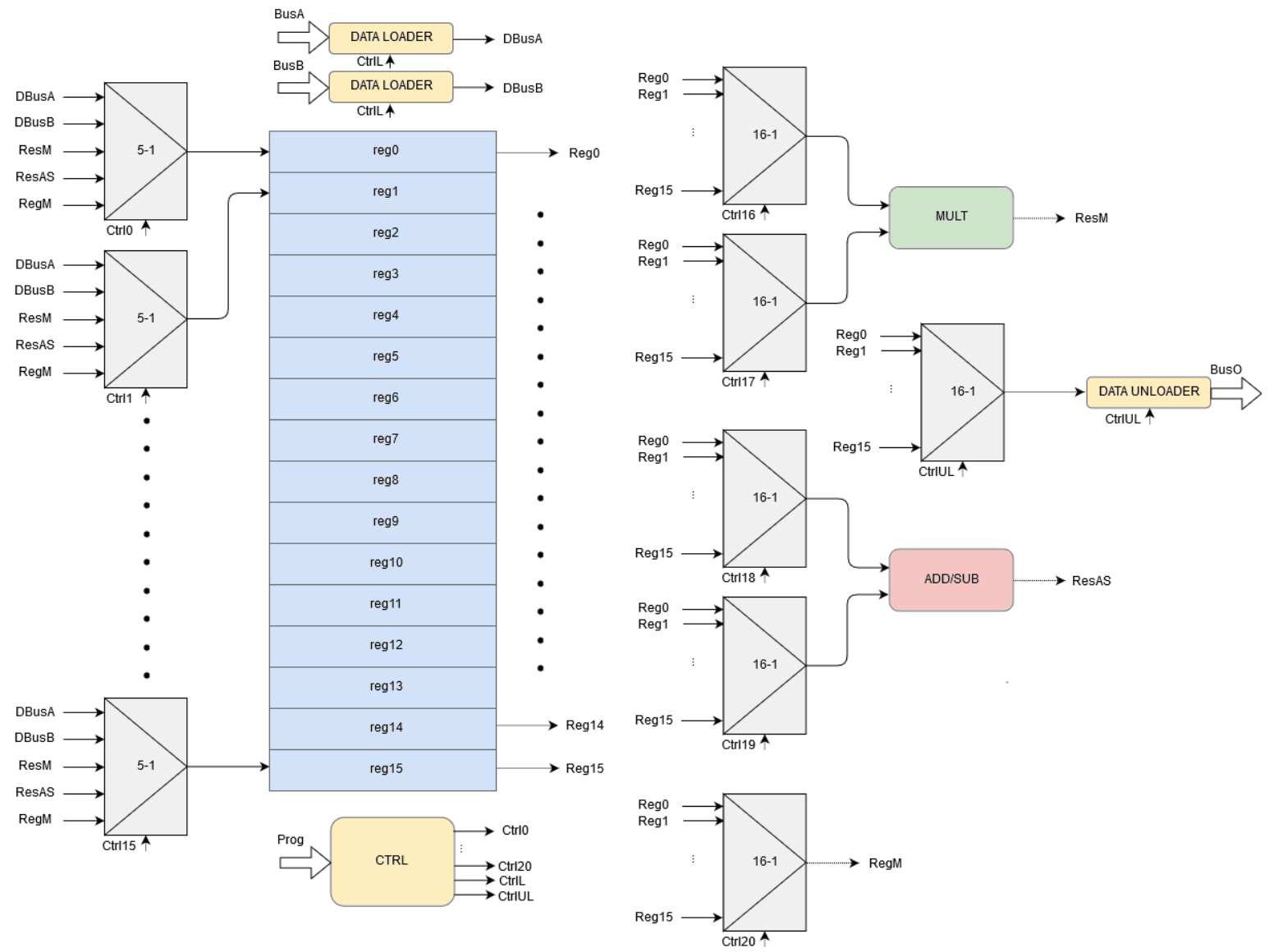

Figure 3. Overall diagram of developed MPA coprocessor (MULT, ADD/SUB and CTRL denote respectively multiplication, adder/subtractor and control units).

The instruction set includes fundamental arithmetic operations (i.e., addition, subtraction, multiplication) as well as data loading instructions. The coprocessor design can be extended with other operations (e.g., bit manipulation instructions) depending on the final user needs. The basic instruction set is presented in Table 1. 
Table 1. Basic instruction set of developed MPA coprocessor.

\begin{tabular}{|c|c|}
\hline Instruction & Result \\
\hline loaa regX & $\operatorname{reg} X=$ data (busA) \\
\hline loab regX & regX = data (busB) \\
\hline loaab regX, regY & $\operatorname{reg} X=$ data (busA) and $\operatorname{reg} Y=$ data (busB) \\
\hline unl regX & data $($ busO) $=\operatorname{regX}$ \\
\hline mult regX, regY, regZ & $\operatorname{reg} Z=\operatorname{reg} X * \operatorname{reg} Y$ \\
\hline add regX, regY, regZ & $\operatorname{reg} Z=\operatorname{reg} X+\operatorname{reg} Y$ \\
\hline sub regX, regY, regZ & $\operatorname{reg} Z=\operatorname{reg} X-\operatorname{reg} Y$ \\
\hline
\end{tabular}

regX, regY, regZ denote any of 16 registers of length $32 \mathrm{kbits}$. busA, busB, busO denote respectively bus A, bus $B$ and output bus.

Instructions loaa, loab, loaab load data to registers from data buses A and B. Instruction unl transfers the value in a register to host CPU using the output data bus. Instructions mult, add, sub are the arithmetic operations of multiplication, addition and subtraction. These instructions are similar to standard processor arithmetic instructions. However, the coprocessor does not emulate MPA operations calling external library functions. Hence, it does not need to perform program jumps and memory operations (e.g., when the size of computation result is larger than the allocated memory) like standard CPU with the MPA software library. However, when the size of the result of MPA operations is larger than $32 \mathrm{kbits}$, there is an overflow, which has to be taken into account, usually during the code development.

Although the proposed MPA coprocessor is presented as operating under the control of host CPU, it can also be implemented in FPGA hardware as a self-contained, programmable device with its own instruction set and data communication channels. That is, although the coprocessor can be managed by host CPU, it only need streams of instructions and data. Hence, the proposed MPA coprocessor can be a valuable solution for the acceleration of MPA computations, not only within SoC solutions for embedded systems but also in other hardware realisations, e.g., FPGA PCIe accelerator cards. Depending on required processing throughput, multiple instances of the MPA coprocessor can still be implemented within a single chip. This flexibility is a vitally important feature of the presented design. Furthermore, due to the implementation in FPGA, the presented MPA coprocessor is highly energy efficient.

\subsection{Multiplier Unit}

Figure 4 shows a block diagram of the multiplier unit. It executes multiplication operations on streamed data of absolute-value operands with the limb size set to 64 bits.

The sign of the result of the multiplication is determined in the control unit. The 64-bit bus pi_data connects each of physical registers with the multiplier. The OPER_SWITCHBOX module selects data in registers to be operands for multiplication operations. Data entering the multiplier are organized by reading from the registers in an appropriate order, so as to obtain the final result ordered from the least-significant to the most-significant limb in the shortest possible time. Then, the MULT_64 module executes the multiplication operations of two 64 bit operands based on the basecase algorithm [29] to give a 128 bit result. Hence, a series of partial results is obtained (each with the length set to two limbs), which are transferred to two MULT_ACC_IP_64 accumulators, each of a single-limb size, with the use of the MULT_DIVERSION demultiplexer. When the currently calculated limb of the result is ready in the MULT_ACC_IP_64 module, it goes through the multiplexer MULT_MUX to the adder MULT_PART_ADDER. Alongside this, the carry is transferred to the next limb. This adder is responsible for adding the limbs with the corresponding carry transferred from the previous limb as well as returning a single limb result and a carry. This data goes to the MULT_CARRY_ADD module, which is an adder of the number and the carry, which allows for getting the final result.

In addition to the described modules, there are two local control modules that form control signals. The MULT_CTRL module is responsible for reading information from the main control unit, decoding 
it and starting the multiplication process. The MULT_SYNC module is responsible for forming control signals so as to enable proper control (at the right time) of modules that process useful data.

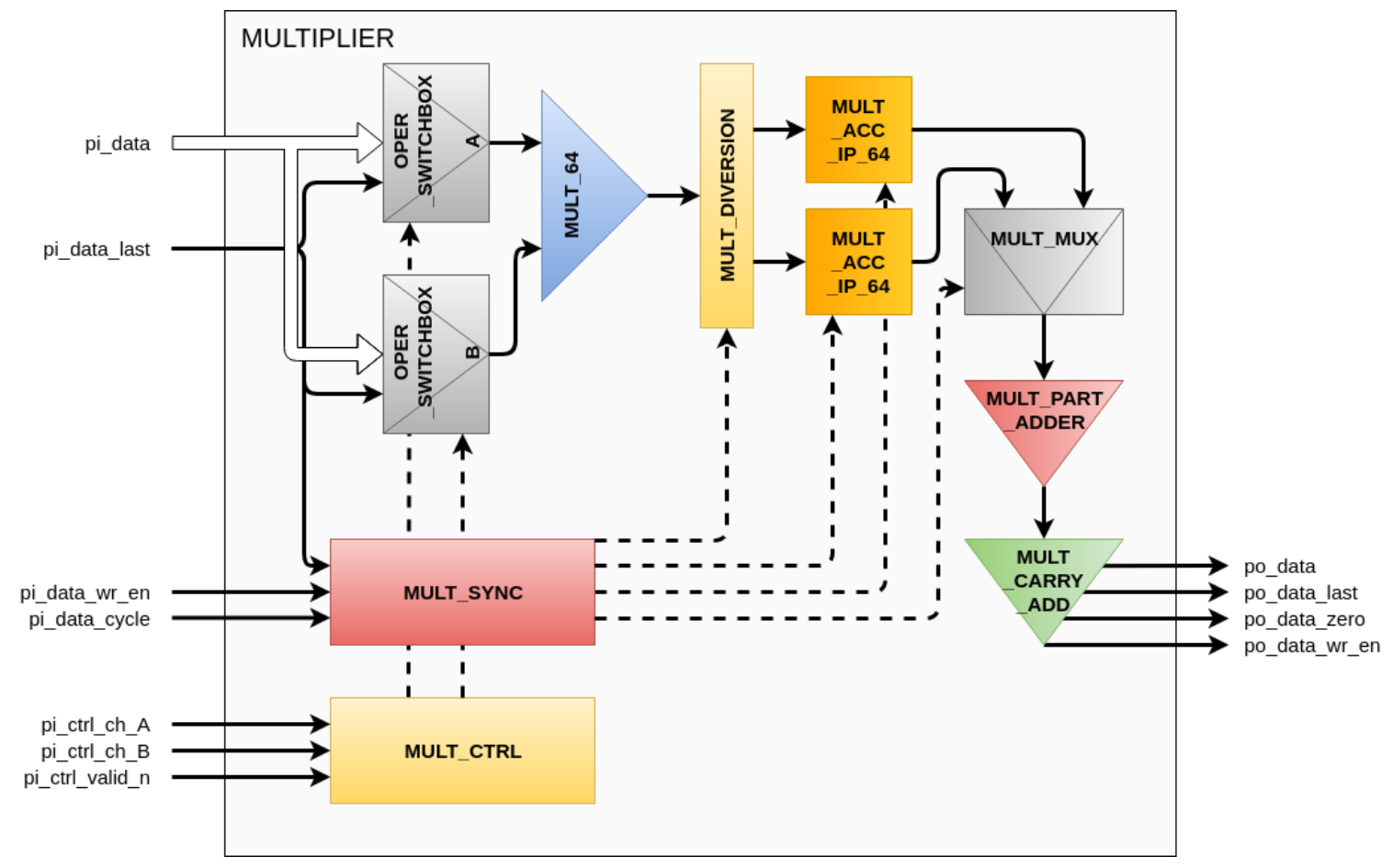

Figure 4. Block diagram of multiplier unit.

\subsection{Adder-Subtractor Unit}

The adder-subtractor unit employs the sign-magnitude representation only for operands and results [24]. Figure 5 presents its block diagram. Each of the ADDER_SUB and ADDER_ADD_SUB blocks consist of two DSP48E1 modules [30] connected to form 64-bit precision modules enabling addition and subtraction. These blocks generate a series of limbs with single-bit carries which are transferred with the use of dedicated lines for DSP block communication.

The unit simultaneously computes two results, i.e., $|\mathrm{B}|-|\mathrm{A}|$ and $|\mathrm{A}|+|\mathrm{B}|$ in parallel using ADDER_SUB and ADDER_ADD_SUB blocks in the case of addition and $|\mathrm{B}|-|\mathrm{A}|$ and $|\mathrm{A}|-|\mathrm{B}|$ in the case of subtraction. There is no quick and easy way to determine in advance which operand is greater, so the sign of the result is known only at the end of the addition/subtraction operation. However, the result can be a negative number in two's complement representation. In this case, the result would have to be converted to the sign-magnitude representation which doubles the processing time, i.e., the bits of the number would have to be inverted and one added to it. Therefore, a double processing pipeline is used and operations are executed on absolute values of operands as described above. In this method, both results are stored in registers (target logical register and auxiliary shadow register) and finally one of them is taken as a valid result with a sign bit adjusted accordingly. Based on the signs of input operands as well as both results of computations, the correct value is taken whose two's complement representation is the same as the magnitude of the result in the sign-magnitude representation. For some cases, the logical number associated with the register of the addition/subtraction output interchanges with the number of the shadow register. Table 2 presents the truth table for the addition operation, allowing us to correctly set the sign of the result as well as the register storing a correct magnitude of the result. Table 3 presents the truth table for the subtraction operation, allowing us to correctly set the sign of the result as well as the register storing a correct magnitude of the result. Such logic functions are implemented within the designed digital circuit. Our design avoids the conversion of addition and subtraction results from two's complement 
representation to the sign-magnitude representation but requires increased resources in terms of logic cells.

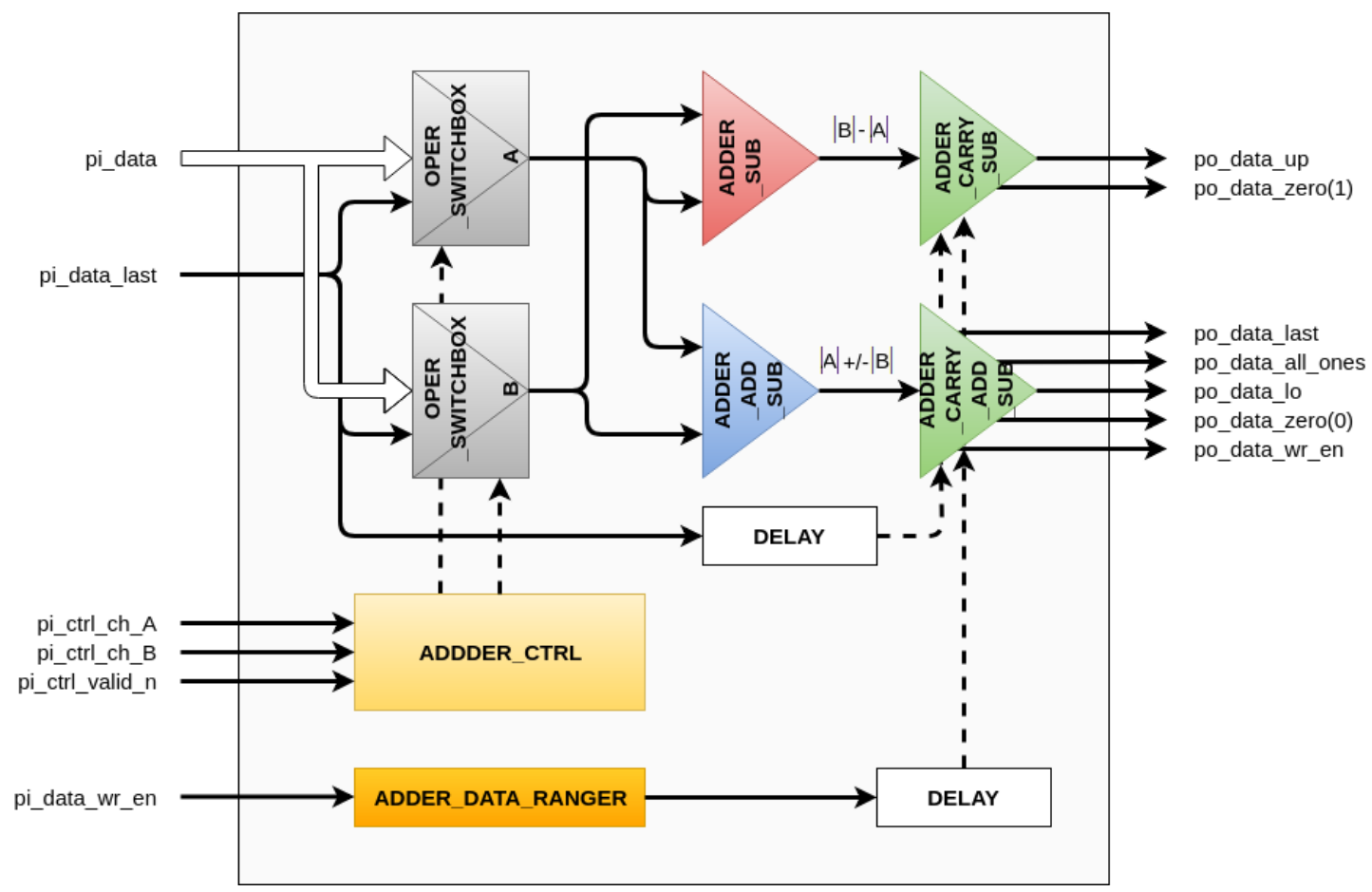

Figure 5. Block diagram of adder-subtractor unit.

Table 2. Truth table allowing us to adjust sign and take corect register as result of addition [24].

\begin{tabular}{|c|c|c|c|c|c|c|c|c|}
\hline$|A|$ vs. $|B|$ & A Sign & B Sign & Example & $\begin{array}{l}\text { Correct } \\
\text { Sign }\end{array}$ & Operations & $\begin{array}{l}\text { Result } \\
\text { Sign }\end{array}$ & $\begin{array}{l}\text { Result } \\
\text { Taken }\end{array}$ & $\begin{array}{l}\text { Sign } \\
\text { Change }\end{array}$ \\
\hline$|A|>|B|$ & $A \geqslant 0$ & $B \geqslant 0$ & $5+2=7$ & + & $\begin{array}{l}\text { ADDER_ADD_SUB: }|A|+|B| \\
\text { ADDER_SUB: }|\mathrm{B}|-|\mathrm{A}|\end{array}$ & $\begin{array}{l}+ \\
-\end{array}$ & $\begin{array}{l}\checkmark \\
\times\end{array}$ & $\times$ \\
\hline$|A|>|B|$ & $A \geqslant 0$ & $\mathrm{~B}<0$ & $5+(-2)=3$ & + & $\begin{array}{l}\text { ADDER_ADD_SUB: }|A|-|B| \\
\text { ADDER_SUB: }|\mathrm{B}|-|\mathrm{A}|\end{array}$ & $\begin{array}{l}+ \\
-\end{array}$ & $\begin{array}{l}\checkmark \\
\times\end{array}$ & $\times$ \\
\hline$|A|>|B|$ & $\mathrm{A}<0$ & $B \geqslant 0$ & $-5+2=-3$ & - & $\begin{array}{l}\text { ADDER_ADD_SUB: }|A|-|B| \\
\text { ADDER_SUB: }|\mathrm{B}|-|\mathrm{A}|\end{array}$ & $\begin{array}{l}+ \\
-\end{array}$ & $\begin{array}{l}\checkmark \\
\times\end{array}$ & $\checkmark$ \\
\hline$|A|>|B|$ & $\mathrm{A}<0$ & $\mathrm{~B}<0$ & $-5+(-2)=-7$ & - & $\begin{array}{l}\text { ADDER_ADD_SUB: }|\mathrm{A}|+|\mathrm{B}| \\
\text { ADDER_SUB: }|\mathrm{B}|-|\mathrm{A}|\end{array}$ & $\begin{array}{l}+ \\
-\end{array}$ & $\begin{array}{l}\checkmark \\
\times\end{array}$ & $\checkmark$ \\
\hline$|A|=|B|$ & $A \geqslant 0$ & $B \geqslant 0$ & $3+3=6$ & + & $\begin{array}{l}\text { ADDER_ADD_SUB: }|A|+|B| \\
\text { ADDER_SUB: }|\mathrm{B}|-|\mathrm{A}|=0\end{array}$ & $\begin{array}{l}+ \\
+\end{array}$ & $\begin{array}{l}\checkmark \\
\times\end{array}$ & $\times$ \\
\hline$|A|=|B|$ & $A \geqslant 0$ & $\mathrm{~B}<0$ & $3+(-3)=0$ & $+(0)$ & $\begin{array}{l}\text { ADDER_ADD_SUB: }|A|-|B|=0 \\
\text { ADDER_SUB: }|\mathrm{B}|-|\mathrm{A}|=0\end{array}$ & $\begin{array}{l}+ \\
+\end{array}$ & $\begin{array}{l}\checkmark \\
\times\end{array}$ & $\times$ \\
\hline$|A|=|B|$ & $\mathrm{A}<0$ & $B \geqslant 0$ & $-3+3=0$ & $+(0)$ & $\begin{array}{l}\text { ADDER_ADD_SUB: }|A|-|B|=0 \\
\text { ADDER_SUB: }|\mathrm{B}|-|\mathrm{A}|=0\end{array}$ & $\begin{array}{l}+ \\
+\end{array}$ & $\begin{array}{l}\checkmark \\
\times\end{array}$ & $\times$ \\
\hline$|A|=|B|$ & $\mathrm{A}<0$ & $\mathrm{~B}<0$ & $-3+(-3)=-6$ & - & $\begin{array}{l}\text { ADDER_ADD_SUB: }|A|+|B| \\
\text { ADDER_SUB: }|B|-|A|=0\end{array}$ & $\begin{array}{l}+ \\
+\end{array}$ & $\begin{array}{l}\checkmark \\
\times\end{array}$ & $\checkmark$ \\
\hline$|\mathrm{A}|<|\mathrm{B}|$ & $A \geqslant 0$ & $B \geqslant 0$ & $2+5=7$ & + & $\begin{array}{l}\text { ADDER_ADD_SUB: }|A|+|B| \\
\text { ADDER_SUB: }|\mathrm{B}|-|\mathrm{A}|\end{array}$ & $\begin{array}{l}+ \\
+\end{array}$ & $\begin{array}{l}\checkmark \\
\times\end{array}$ & $\times$ \\
\hline$|\mathrm{A}|<|\mathrm{B}|$ & $A \geqslant 0$ & $\mathrm{~B}<0$ & $2+(-5)=-3$ & - & $\begin{array}{l}\text { ADDER_ADD_SUB: }|A|-|B| \\
\text { ADDER_SUB: }|\mathrm{B}|-|\mathrm{A}|\end{array}$ & $\begin{array}{l}- \\
+\end{array}$ & $\begin{array}{l}\times \\
\checkmark\end{array}$ & $\checkmark$ \\
\hline$|A|<|B|$ & $\mathrm{A}<0$ & $B \geqslant 0$ & $-2+5=3$ & + & $\begin{array}{l}\text { ADDER_ADD_SUB: }|\mathrm{A}|-|\mathrm{B}| \\
\text { ADDER_SUB: }|\mathrm{B}|-|\mathrm{A}|\end{array}$ & $\begin{array}{l}- \\
+\end{array}$ & $\begin{array}{l}\times \\
\checkmark\end{array}$ & $\times$ \\
\hline$|A|<|B|$ & $\mathrm{A}<0$ & $\mathrm{~B}<0$ & $-2+(-5)=-7$ & - & $\begin{array}{l}\text { ADDER_ADD_SUB: }|A|+|B| \\
\text { ADDER_SUB: }|B|-|A|\end{array}$ & $\begin{array}{l}+ \\
+\end{array}$ & $\begin{array}{l}\checkmark \\
\times\end{array}$ & $\checkmark$ \\
\hline
\end{tabular}


Table 3. Truth table allowing us to adjust sign and take corect register as result of subtraction [24].

\begin{tabular}{|c|c|c|c|c|c|c|c|c|}
\hline$|A|$ vs. $|\mathrm{B}|$ & A Sign & B Sign & Example & $\begin{array}{l}\text { Correct } \\
\text { Sign }\end{array}$ & Operations & $\begin{array}{l}\text { Result } \\
\text { Sign }\end{array}$ & $\begin{array}{l}\text { Result } \\
\text { Taken }\end{array}$ & $\begin{array}{l}\text { Sign } \\
\text { Change }\end{array}$ \\
\hline$|A|>|B|$ & $A \geqslant 0$ & $B \geqslant 0$ & $5-2=3$ & + & $\begin{array}{l}\text { ADDER_ADD_SUB: }|A|-|\mathrm{B}| \\
\text { ADDER_SUB: }|\mathrm{B}|-|\mathrm{A}|\end{array}$ & $\begin{array}{l}+ \\
-\end{array}$ & $\begin{array}{l}\checkmark \\
\times\end{array}$ & $x$ \\
\hline$|A|>|B|$ & $A \geqslant 0$ & $\mathrm{~B}<0$ & $5-(-2)=7$ & + & $\begin{array}{l}\text { ADDER_ADD_SUB: }|A|+|B| \\
\text { ADDER_SUB: }|\mathrm{B}|-|\mathrm{A}|\end{array}$ & $\begin{array}{l}+ \\
-\end{array}$ & $\begin{array}{l}\checkmark \\
\times\end{array}$ & $x$ \\
\hline$|A|>|B|$ & $\mathrm{A}<0$ & $B \geqslant 0$ & $-5-2=-7$ & - & $\begin{array}{l}\text { ADDER_ADD_SUB: }|\mathrm{A}|+|\mathrm{B}| \\
\text { ADDER_SUB: }|\mathrm{B}|-|\mathrm{A}|\end{array}$ & + & $\begin{array}{l}\checkmark \\
\times\end{array}$ & $\checkmark$ \\
\hline$|A|>|B|$ & $\mathrm{A}<0$ & $\mathrm{~B}<0$ & $-5-(-2)=-3$ & - & $\begin{array}{l}\text { ADDER_ADD_SUB: }|\mathrm{A}|-|\mathrm{B}| \\
\text { ADDER_SUB: }|\mathrm{B}|-|\mathrm{A}|\end{array}$ & $\begin{array}{l}+ \\
-\end{array}$ & $\begin{array}{l}\checkmark \\
\times\end{array}$ & $\checkmark$ \\
\hline$|A|=|B|$ & $A \geqslant 0$ & $B \geqslant 0$ & $3-3=0$ & $+(0)$ & $\begin{array}{l}\text { ADDER_ADD_SUB: }|A|-|\mathrm{B}|=0 \\
\text { ADDER_SUB: }|\mathrm{B}|-|\mathrm{A}|=0\end{array}$ & $\begin{array}{l}+ \\
+\end{array}$ & $\begin{array}{l}\checkmark \\
\times\end{array}$ & $x$ \\
\hline$|A|=|B|$ & $A \geqslant 0$ & $\mathrm{~B}<0$ & $3-(-3)=6$ & + & $\begin{array}{l}\text { ADDER_ADD_SUB: }|\mathrm{A}|+|\mathrm{B}| \\
\text { ADDER_SUB: }|\mathrm{B}|-|\mathrm{A}|=0\end{array}$ & $\begin{array}{l}+ \\
+\end{array}$ & $\begin{array}{l}\checkmark \\
\times\end{array}$ & $x$ \\
\hline$|A|=|B|$ & $\mathrm{A}<0$ & $B \geqslant 0$ & $-3-3=-6$ & - & $\begin{array}{l}\text { ADDER_ADD_SUB: }|A|+|B| \\
\text { ADDER_SUB : }|\mathrm{B}|-|\mathrm{A}|=0\end{array}$ & $\begin{array}{l}+ \\
+\end{array}$ & $\begin{array}{l}\checkmark \\
\times\end{array}$ & $\checkmark$ \\
\hline$|A|=|B|$ & $\mathrm{A}<0$ & $\mathrm{~B}<0$ & $-3-(-3)=0$ & $+(0)$ & $\begin{array}{l}\text { ADDER_ADD_SUB: }|A|-|B|=0 \\
\text { ADDER_SUB: }|\mathrm{B}|-|\mathrm{A}|=0\end{array}$ & $\begin{array}{l}+ \\
+\end{array}$ & $\begin{array}{l}\checkmark \\
\times\end{array}$ & $x$ \\
\hline$|A|<|B|$ & $A \geqslant 0$ & $B \geqslant 0$ & $2-5=-3$ & - & $\begin{array}{l}\text { ADDER_ADD_SUB: }|\mathrm{A}|-|\mathrm{B}| \\
\text { ADDER_SUB: }|\mathrm{B}|-|\mathrm{A}|\end{array}$ & $\begin{array}{l}- \\
+\end{array}$ & $\begin{array}{l}x \\
x\end{array}$ & $\checkmark$ \\
\hline$|\mathrm{A}|<|\mathrm{B}|$ & $A \geqslant 0$ & $\mathrm{~B}<0$ & $2-(-5)=7$ & + & $\begin{array}{l}\text { ADDER_ADD_SUB: }|A|+|B| \\
\text { ADDER_SUB: }|\mathrm{B}|-|\mathrm{A}|\end{array}$ & $\begin{array}{l}+ \\
+\end{array}$ & $\begin{array}{l}\checkmark \\
\times\end{array}$ & $x$ \\
\hline$|\mathrm{A}|<|\mathrm{B}|$ & $\mathrm{A}<0$ & $B \geqslant 0$ & $-2-5=-7$ & - & $\begin{array}{l}\text { ADDER_ADD_SUB: }|A|+|B| \\
\text { ADDER_SUB: }|\mathrm{B}|-|\mathrm{A}|\end{array}$ & $\begin{array}{l}+ \\
+\end{array}$ & $\begin{array}{l}\checkmark \\
\times\end{array}$ & $\checkmark$ \\
\hline$|\mathrm{A}|<|\mathrm{B}|$ & $\mathrm{A}<0$ & $\mathrm{~B}<0$ & $-2-(-5)=3$ & + & $\begin{array}{l}\text { ADDER_ADD_SUB: }|\mathrm{A}|-|\mathrm{B}| \\
\text { ADDER_SUB: }|\mathrm{B}|-|\mathrm{A}|\end{array}$ & $\begin{array}{l}- \\
+\end{array}$ & $\begin{array}{l}\times \\
\checkmark\end{array}$ & $x$ \\
\hline
\end{tabular}

\section{Hardware Implementation}

Figure 6 presents the IP block of the MPA coprocessor, which targets Zynq-7000 Xilinx SoC (FPGA combined with ARM Cortex A9 processor). The correctness of computations and speedup ratios are evaluated based on Aldec TySOM-1 board [27].

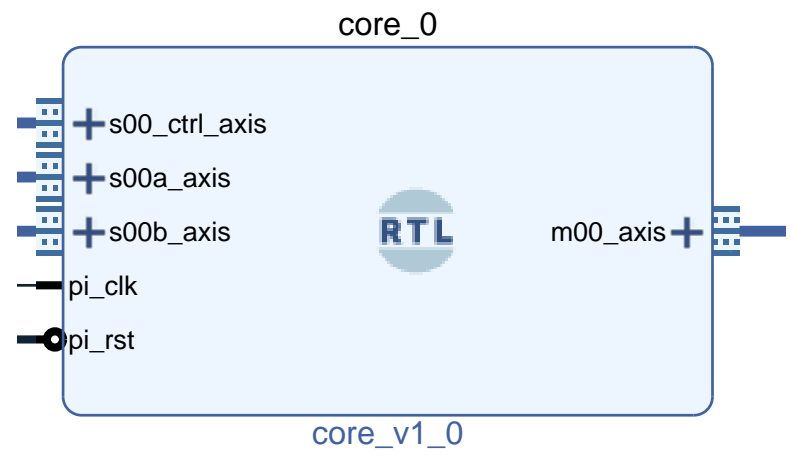

Figure 6. RTL block of MPA coprocessor in Xilinx Vivado tool.

The block of the MPA coprocessor includes three AXI Stream (AXIS) interfaces configured as slave ports to receive data and instructions as well as a single AXIS interface configured as a master port to return results of MPA computations. However, the host processor (ARM Cortex A9 core) communicates with FPGA using an AXI4 interface. Hence, the connection between ARM Cortex A9 cores and the MPA coprocessor in FPGA is implemented based on the AXI interconnect block, dedicated AXI4-AXIS converters and FIFO blocks for data storage and buffering. This solution ensures the continuous flow of data to the MPA coprocessor. Furthermore, the ARM Cortex A9 processor can send new data for computations to the FIFO buffers and does not have to wait for finish of MPA computations (i.e., it is non-blocking operation). Figure 7 shows the data-flow diagram for the operation of data writing to the MPA coprocessor, where orange wires denote employed AXI connections. The host processor controls FIFO buffers connected by AXIS slave interfaces with the use of AXI master port (GP0). 


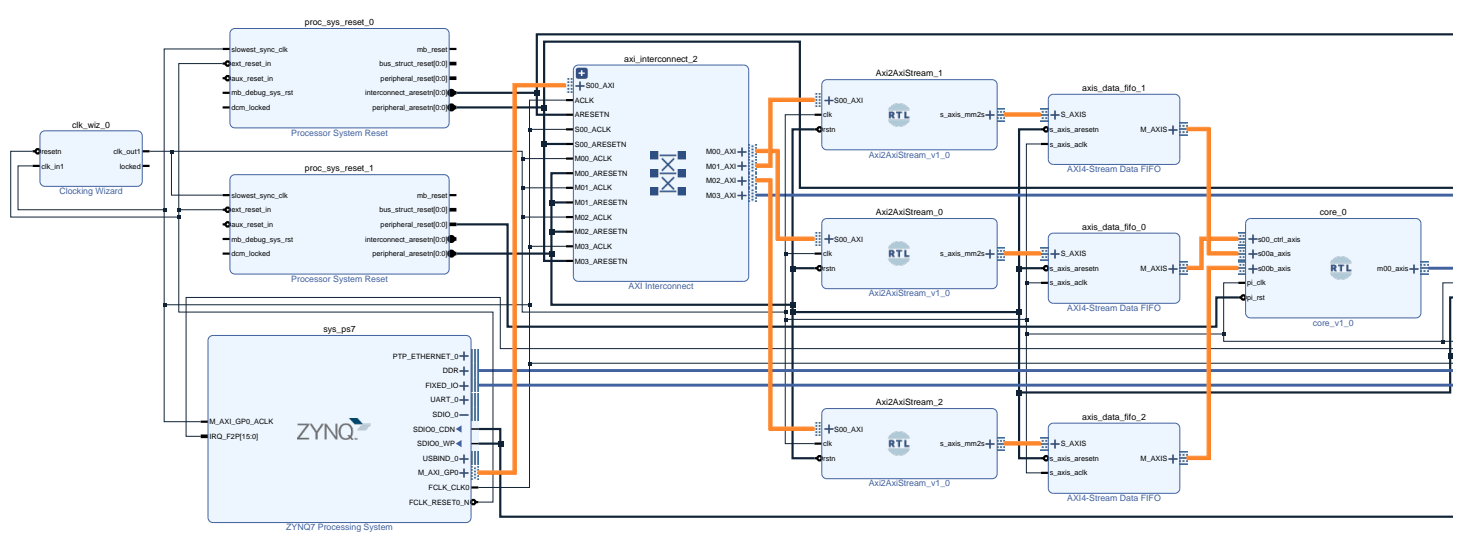

Figure 7. Data-flow diagram for operation of data-writing to MPA coprocessor.

The final result of the computations is sent by the MPA coprocessor to BRAM through the AXIS master interface. For this purpose, the AXIS-AXI4 converter and the AXI interconnect block are used to store data and share it with ARM Cortex A9. Figure 8 shows the data-flow diagram for the connection between the coprocessor and BRAM, where orange wires denote active AXI connections.

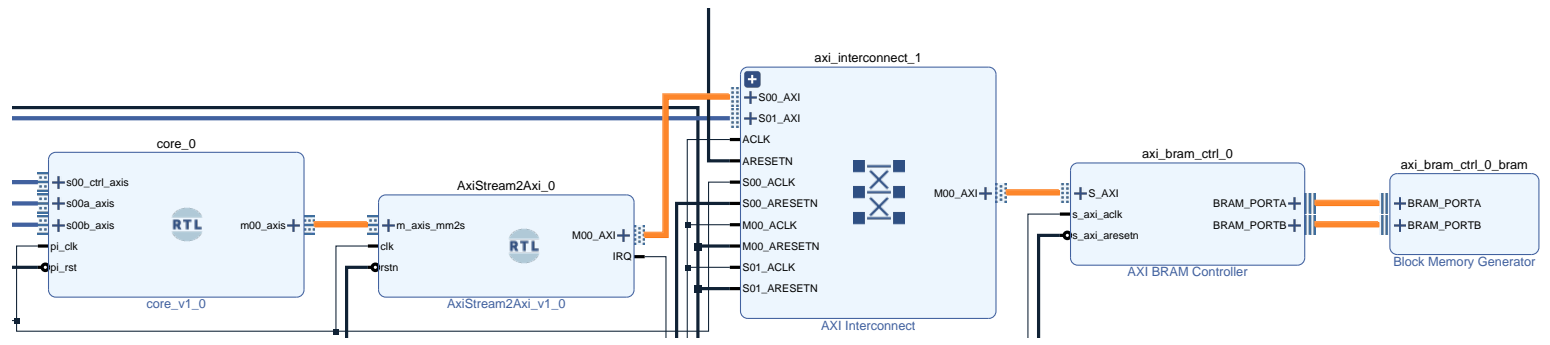

Figure 8. Data-flow diagram for data-writing operation to block random access memory (BRAM) from MPA coprocessor.

The MPA coprocessor is implemented in FPGA with the clock frequency equal to $400 \mathrm{MHz}$ (less than the physical limit of $458 \mathrm{MHz}$ for this FPGA). The time of the code execution on the MPA coprocessor is measured with the use of an additional timer triggered by the rising edge of the clock at the beginning of the execution of the first code instruction. The end of computations is triggered by the first transfer of resulting data (signal TVALID $=1$ on AXIS master interface). The results of software simulation and hardware verification are always consistent in terms of the number of clock cycles in executed operations. Of course, obtained speedups of the MPA coprocessor against CPU depend on the clock frequency. Such a design requires currently hardware resources as in Table 4, where the resources are presented for the MPA coprocessor alone and with the infrastructure for communication with host CPU. With the use of resources available on Zynq-7000 SoC, the implementation of six MPA cores is possible in this chip assuming $80 \%$ utilization of FPGA resources.

The Aldec TySOM-1 board operates under the control of a Linux real-time operating system (RTOS) with a hardware application layer (HAL) that supports AXI4 interface. The application is developed based on HAL functions. It transfers a code to the MPA coprocessor and waits for the result. However, the times measured in benchmarks do not include the read latency stemming from data transfers between BRAM and the host processor through M_AXI_GP0 port. It is verified that the communication between the MPA and host processors using the AXI interconnect does not introduce significant overhead to the benchmarks presented below. That is, the measured time for writing 64 kbits of data (two MPA numbers of the maximal size) to the MPA coprocessor is equal to $0.18 \mu \mathrm{s}$. Then, the measured time for reading $64 \mathrm{kbits}$ of data from the MPA coprocessor is equal to $0.16 \mu \mathrm{s}$. Hence, one can conclude when it is reasonable to use the proposed MPA coprocessor implemented on 
Zynq-7000 SoC. That is, the communication overhead between CPU and FPGA has to be negligible in comparison with the computation time on CPU using the MPA software library.

Table 4. Resources required for implementation of MPA coprocessor.

\begin{tabular}{lcccccc}
\hline MPA Coprocessor & \multicolumn{3}{c}{ Alone } & \multicolumn{3}{c}{ With Infrastructure } \\
\hline Resources & Used & Available & Utilization (\%) & Used & Available & Utilization (\%) \\
\hline slice LUTs & 8745 & 78,600 & 11.13 & 15,735 & 78,600 & 20.02 \\
$\quad$ LUT as logic & 8673 & 78,600 & 11.03 & 14,238 & 78,600 & 18.11 \\
LUT as memory & 72 & 26,600 & 0.27 & 1497 & 26,600 & 5.63 \\
slice registers & 16,249 & 157,200 & 10.34 & 27,708 & 157,200 & 17.63 \\
$\quad$ register as flip flop & 16,249 & 157,200 & 10.34 & 27,705 & 157,200 & 17.62 \\
register as latch & 0 & 157,200 & 0 & 3 & 157,200 & $<0.01$ \\
F7 muxes & 482 & 39,300 & 1.23 & 813 & 39,300 & 2.07 \\
F8 muxes & 66 & 19,650 & 0.34 & 98 & 19,650 & 0.50 \\
block RAM tile & 18 & 265 & 6.79 & 27 & 265 & 10.19 \\
RAMB36/FIFO & 18 & 265 & 6.79 & 27 & 265 & 10.19 \\
DSPs & 34 & 400 & 8.50 & 34 & 400 & 8.50 \\
\hline
\end{tabular}

\section{Results}

The coprocessor is benchmarked in factorial, exponentiation and DGF computations, using codes written for this purpose. The same computations are executed with the use of the GMP library [29], which allows one to compute the same results as the MPA coprocessor using ARM Cortex A9 on TySOM-1 @ $650 \mathrm{MHz}$. The performance of the developed MPA coprocessor is measured in hardware as well as estimated based on simulation results in the design software [31]. Results from the hardware execution and the simulation are always the same.

\subsection{Computations of Factorial}

In Table 5, listing of the code for factorial computations is presented for $n=4$ [23]. In lines 1 and 2 , reg0, reg2, reg3 are loaded with initial data equal to 1 . Then (line 3), value of reg4 is obtained by adding reg2 and reg3 (reg4 =2). In the next line (line 4 ), reg4 is multiplied by reg0 and result is stored in reg1 (reg1 = 2). Then (line 5), value of reg2 is obtained by adding reg4 and reg3 (reg2 = 3). In the next line (line 6), reg2 is multiplied by reg1 and result is stored in reg0 (reg0 $=6)$. Then (line 7), value of reg4 is obtained by adding reg2 and reg3 (reg4 =4). In the next line (line 8 ), reg4 is multiplied by reg0 and result is stored in reg1 (reg1 $=24)$. Finally, the MPA number in reg1 is unloaded in line 9.

Table 5. Listing of code for factorial computations $(n=4)$ [23].

\begin{tabular}{cl}
\hline Line & Instruction \\
\hline 1 & loaab reg0, reg2; \\
2 & loaa reg3; \\
3 & add reg2, reg3, reg4; \\
4 & mult reg4, reg0, reg1; \\
5 & add reg4, reg3, reg2; \\
6 & mult reg2, reg1, reg0; \\
7 & add reg2, reg3, reg4; \\
8 & mult reg4, reg0, reg1; \\
9 & unl reg1; \\
\hline reg0, reg2, reg3 are initially loaded with 1.
\end{tabular}

In Figure 9, measured runtimes for the MPA coprocessor and the ARM Cortex A9 core are presented for factorial ( $n$ !) computations for $n$ varying in the range approaching $n=1000$.

To compute $n$ ! for $n=1000$, a single core of an ARM Cortex A9 processor needs $1092 \mu$ s whereas the MPA coprocessor needs $324 \mu \mathrm{s}$. In this test, the developed MPA coprocessor is 3.37 times faster than a core of CPU within the same SoC. 


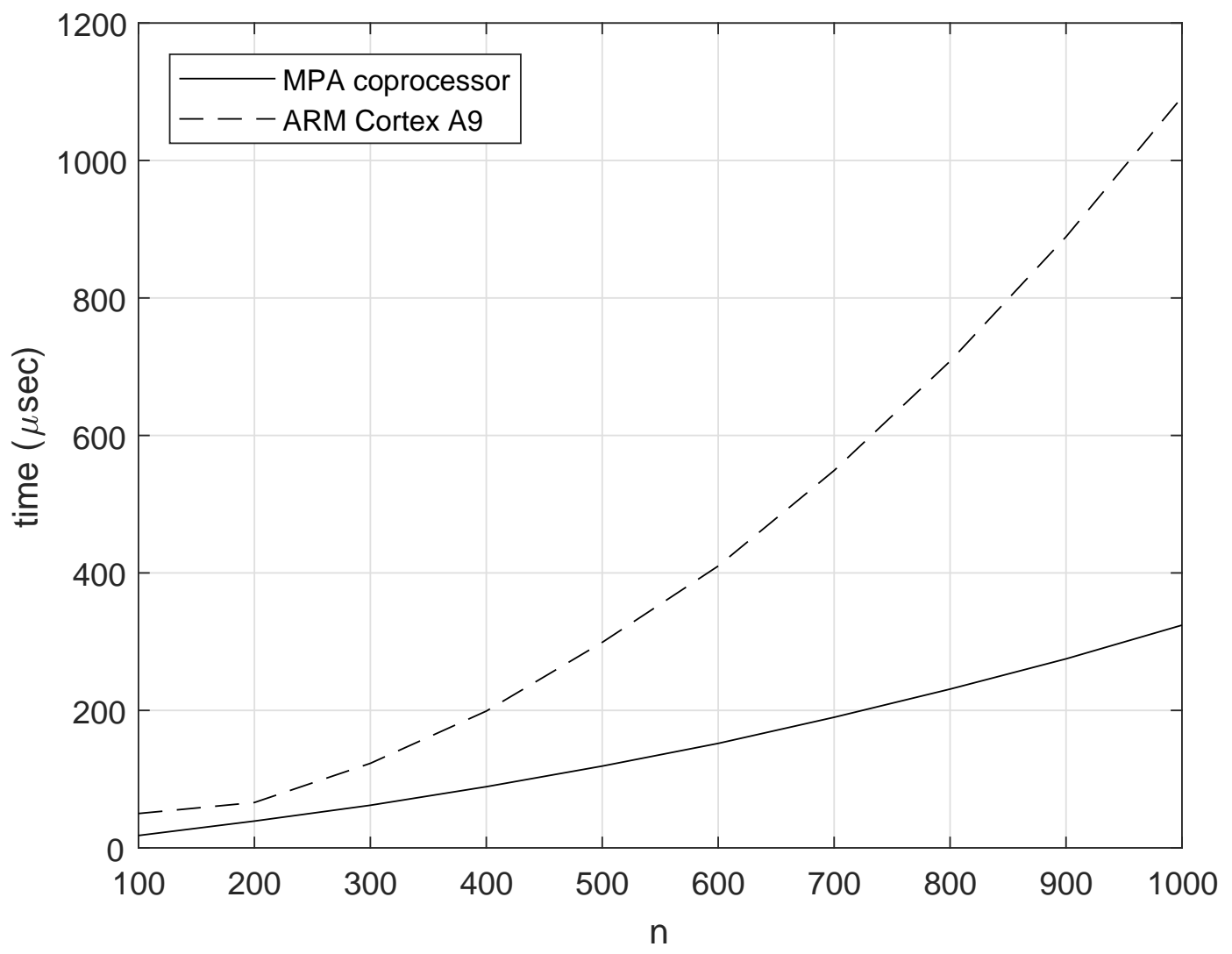

Figure 9. Runtimes for factorial computations ( $n !)$.

\subsection{Computations of Exponentiation}

In Table 6, listing of the code for $n$-th power of $n$ computations is presented for $n=4$. In line 1 , reg0 and reg1 are loaded with initial data equal to $n=4$. Then (line 2), value of reg2 is obtained by multiplying reg0 and reg1 (reg0 = reg1 = $n=4)$. In the next line (line 3$)$, reg2 is multiplied by reg 1 and result is stored in reg0 $(\mathrm{reg} 2=16)$. Then (line 4$)$, value of reg2 is obtained by multiplying reg0 and reg1 (reg2 = 256). Finally, value of reg2 is unloaded in line 5.

Table 6. Listing of code for $n$-th power of $n$ computations $(n=4)$.

\begin{tabular}{cl}
\hline Line & Instruction \\
\hline 1 & loaab reg0, reg1; \\
2 & mult reg0, reg1, reg2; \\
3 & mult reg2, reg1, reg0; \\
4 & mult reg0, reg1, reg2; \\
5 & unl reg2; \\
reg0, reg1 are initially loaded with $n$.
\end{tabular}

In Figure 10, measured runtimes for the MPA coprocessor and the ARM Cortex A9 core are presented for $n$-th power of $n\left(n^{n}\right)$ computations for $n$ varying in the range approaching $n=1000$.

To compute $n^{n}$ for $n=1000$, a single core of an ARM Cortex A9 processor needs $1267 \mu$ s whereas the MPA coprocessor needs $366 \mu \mathrm{s}$. In this test, the developed MPA coprocessor is 3.46 times faster than a single core of ARM A9 processor within the same SoC. 


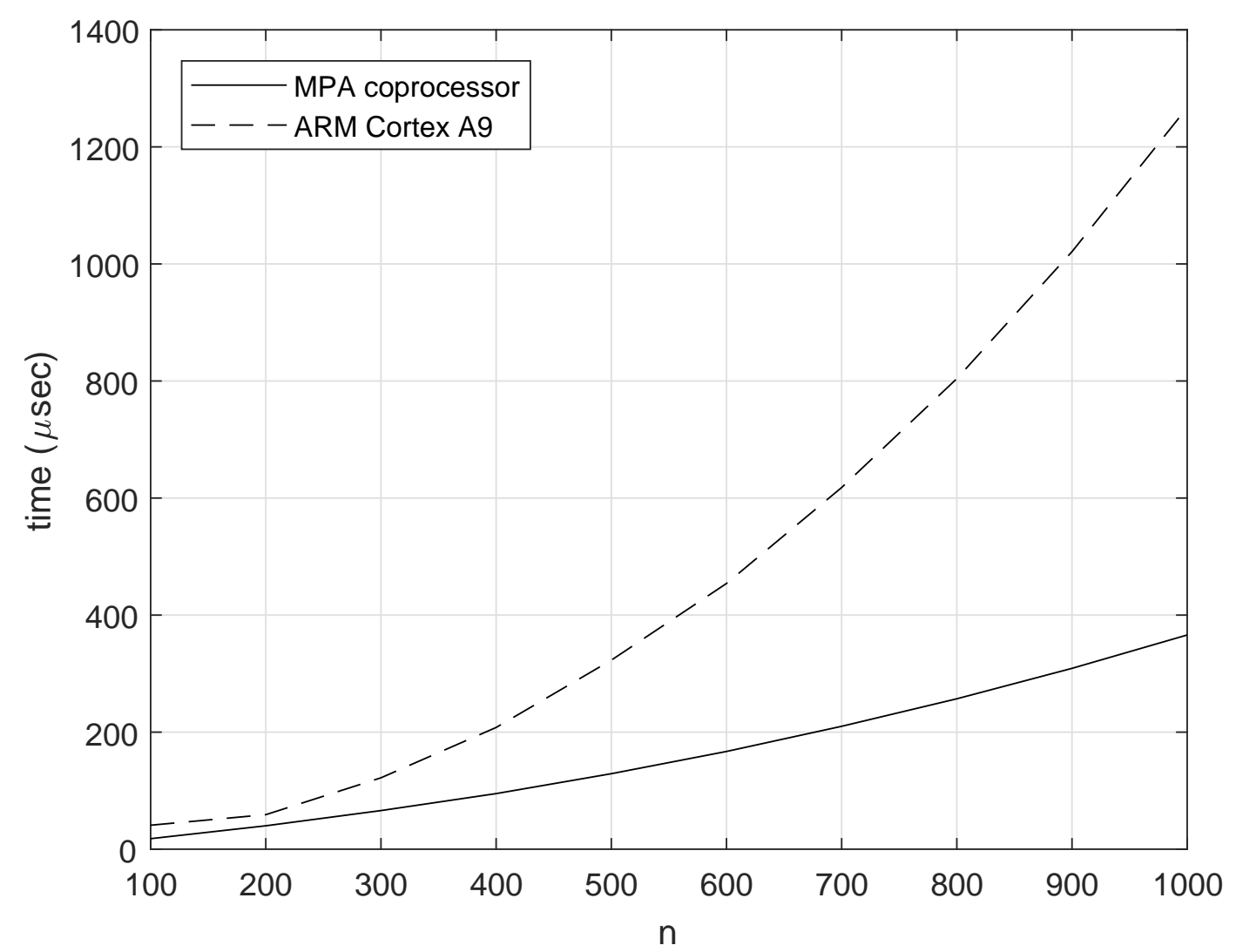

Figure 10. Runtimes for $n$-th power of $n$ computations $\left(n^{n}\right)$.

\subsection{Computations of DGF}

The MPA coprocessor is benchmarked in computations of the one-dimmensional (1-D) scalar DGF in electromagnetics [2-5,32]. This function allows one to represent 1-D finite-difference time-domain (FDTD) update equations [32,33] with the use of the convolution formulation

$$
\left[\begin{array}{c}
E_{k}^{n} \\
H_{k}^{n}
\end{array}\right]=\sum_{n^{\prime} k^{\prime}}\left[\begin{array}{cc}
G_{e e} k-n^{\prime} & G_{e h-k^{\prime}}^{n-n^{\prime}} \\
G_{h e} n-n^{\prime} & G_{h k^{\prime}}^{n-n^{\prime}} \\
k-k^{\prime}
\end{array}\right] \cdot\left[\begin{array}{c}
J_{k^{\prime}}^{n^{\prime}} \\
M_{k^{\prime}}^{n^{\prime}}
\end{array}\right]
$$

where $E$ and $H$ denote, respectively, electric- and magnetic-field vectors, $J$ and $M$ denote, respectively, electric- and magnetic-current source vectors, and $n$ and $k$ denote, respectively, temporal and spatial indices of the 1-D FDTD grid. $G_{e e}, G_{e h}, G_{h e}, G_{h h}$ functions can be obtained for a free space as follows:

$$
\begin{aligned}
G_{e e k}^{n} & =-\frac{\Delta t}{\epsilon_{0}}\left(g_{k}^{n}-g_{k}^{n-1}\right) \\
G_{e h k}^{n} & =\Delta z \gamma^{2}\left(g_{k}^{n}-g_{k-1}^{n}\right) \\
G_{h e k} & =\Delta z \gamma^{2}\left(g_{k+1}^{n}-g_{k}^{n}\right) \\
G_{h h k}{ }^{n} & =-\frac{\Delta t}{\mu_{0}}\left(g_{k}^{n+1}-g_{k}^{n}\right)
\end{aligned}
$$

where $\epsilon_{0}$ and $\mu_{0}$ denote, respectively, permittivity and permeability of the free space, and $\Delta t$ and $\Delta z$ denote, respectively, temporal- and spatial-step sizes. 1-D scalar DGF is computed with the use of the following formula:

$$
g_{k}^{n}=\sum_{m=k}^{n-1} \gamma^{2 m}\left(\begin{array}{c}
m+n \\
2 m+1
\end{array}\right)\left(\begin{array}{c}
2 m \\
m+k
\end{array}\right)(-1)^{m+k}
$$


where $\gamma=\frac{c \Delta t}{\Delta z}\left(c=1 / \sqrt{\mu_{0} \epsilon_{0}}\right)$ denotes the Courant number that must be less than or equal to one $(\gamma \leq 1)$ to guarantee the stability of computations. The implementation of (3) in software requires MPA [2]. Let us assume $\gamma=1$ because a simple formula for scalar DGF computations (provable by the mathematical induction [32]) exists in this case

$$
g_{k}^{n}= \begin{cases}0, & n \leq k \\ 0, & n>k \text { and }(n+k) \text { even } . \\ 1, & n>k \text { and }(n+k) \text { odd }\end{cases}
$$

Hence, 1-D scalar DGF is computed in our benchmark with the use of (3) for $\gamma=1$ and the correctness of the computations is verified with the use of (4). In the codes, binomial coefficients are loaded from memory in order resulting from the sum in (3). Thus, only the computational throughput in multiplication and addition/subtraction of MPA numbers is measured in this benchmark.

In Table 7, listing of the code for DGF computations is presented for $n=4$ and $k=0$. In line 1 , reg4 is loaded with 0 . Then (line 2), reg0 and reg1 are loaded with 4 and 1 , respectively. Then (line $3)$, value of reg2 is obtained by multiplying reg0 and reg1. In the next line (line 4), reg4 is added to reg2 and result is stored in reg3. Then (line 5), reg0 and reg1 are loaded with 10 and 2, respectively. Then (line 6), value of reg2 is obtained by multiplying reg0 and reg1. In the next line (line 7), reg2 is subtracted from reg3 and result is stored in reg4. Then (line 8), reg0 and reg1 are both loaded with 6 . Then (line 9), value of reg2 is obtained by multiplying reg0 and reg1. In the next line (line 10), reg4 is added to reg2 and the result is stored in reg3. Then (line 11), reg0 and reg1 are loaded with 1 and 20, respectively. Then (line 12), value of reg2 is obtained by multiplying reg0 and reg1. In the next line (line 13), reg2 is subtracted from reg3 and result is stored in reg4 (reg4=0). Finally, value of reg4 is unloaded in line 14 .

Table 7. Listing of code for discrete Green's function (DGF) computations ( $n=4, k=0)$.

\begin{tabular}{ll}
\hline Line & Instruction \\
\hline 1 & loaa reg4; \\
2 & loaab reg0, reg1; \\
3 & mult reg0, reg1, reg2; \\
4 & add reg4, reg2, reg3; \\
5 & loaab reg0, reg1; \\
6 & mult reg0, reg1, reg2; \\
7 & sub reg3, reg2, reg4; \\
8 & loaab reg0, reg1; \\
9 & mult reg0, reg1, reg2; \\
10 & add reg4, reg2, reg3; \\
11 & loaab reg0, reg1; \\
12 & mult reg0, reg1, reg2; \\
13 & sub reg3, reg2, reg4; \\
14 & unl reg4; \\
\hline
\end{tabular}

Line 1: reg4 is loaded with 0 . Line 2: reg0, reg1 are respectively loaded with 4 and 1 . Line 5: reg0, reg1 are respectively loaded with 10 and 2. Line 8: reg0, reg1 are both loaded with 6. Line 11: reg0, reg1 are respectively loaded with 1 and 20.

In Figure 11, measured runtimes for the MPA coprocessor and the ARM Cortex A9 core are presented for $k=0$ and $k=99$ with $n$ varying in the range approaching 2000. To compute DGF for $n=2000$ and $k=0(k=99)$, a single core of ARM A9 processor needs $57,254 \mu \mathrm{s}(56,416 \mu \mathrm{s})$ whereas the MPA coprocessor needs $5529.8 \mu \mathrm{s}(5446.3 \mu \mathrm{s})$. For these parameters, the developed MPA coprocessor is around 10.35 times faster than a single core of ARM A9 processor within the same SoC. For the MPA coprocessor, the DGF computation time can be estimated as

$$
t_{D G F}=A n^{\alpha} f_{c l k}^{-1}
$$


With the use of regression for $n \geq 1000, A=0.0046, \alpha=2.6287$ and $A=0.0028, \alpha=2.6908$ are obtained for $k=0$ and $k=99$, respectively. For the considered design, the clock frequency $f_{c l k}$ is set to $400 \mathrm{MHz}$.

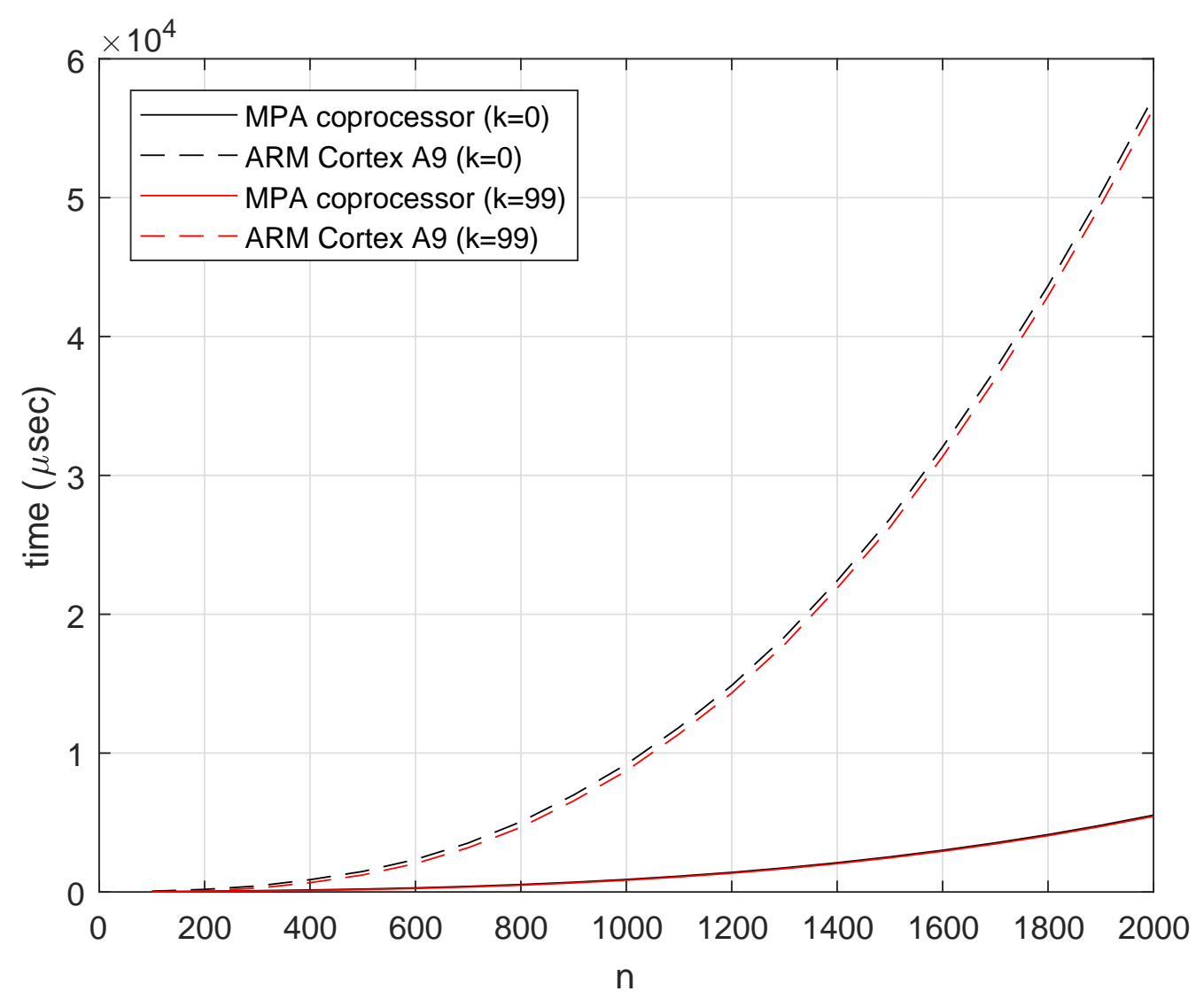

Figure 11. Runtimes for DGF computations. MPA coprocessor results overlap for $k=0$ and $k=99$.

To sum up the results, the developed coprocessor is benchmarked in factorial, exponentiation and DGF computations. In factorial and exponentiation computations, the coprocessor (implemented in FPGA on SoC) can be three times faster than a single CPU core within the same chip. In the task of DGF generation, the MPA coprocessor is more than ten times faster than a single CPU core within the same chip. The obtained difference between the speedups for factorial/exponentiation and DGF computations is due to the structure of the DGF code. The difference depends on the amount of data retrieved from the processor memory. The ARM Cortex A9 processor can perform short computations using L1 cache when code jumps do not exceed its size. This is the case with the ARM Cortex A9 equipped with $32 \mathrm{~KB}$ L1 cache, hence these operations are carried out quickly in factorial/exponentiation computations. When computations make it impossible to enter the pipeline, numerous jumps in memory are executed as in the case of DGF computations. Then, the cache size is insufficient to optimize the code execution and the ARM Cortex A9 performance decreases compared to the MPA coprocessor, which has a dedicated architecture of long registers and pipelined data processing. Hence, the MPA coprocessor does not execute program loops, memory operations and does not call external MPA library functions, which slow down CPU. It is verified in simulation that the ratio of the total execution time of arithmetic operations to the total time of the code execution approaches $99 \%$ for the factorial and exponentiation computations $(n=1000)$. In the DGF computations, this coefficient is equal to $96 \%$ and $98 \%$ for $n=1000$ and $n=2000$, respectively. It confirms the efficient implementation of the data processing in the MPA coprocessor. Hence, further improvements in the coprocessor design can be obtained using faster arithmetic units. However, 
the dedicated coprocessor for integer MPA computations is not as flexible a computational unit as CPU. Furthermore, operand sizes for MPA operations are limited up to $32 \mathrm{kbits}$ in our design. It is worth noticing that ARM cores are hard-core processors, whereas the MPA coprocessor is implemented within the programmable logic. Hence, FPGA within SoC can still be reprogrammed to implement various logic functions, depending upon user needs. Furthermore, it is still possible to implement several MPA cores within SoC, allowing for flexible reconfigurable parallel computing.

\section{Materials and Methods}

The Vivado Design Suite from Xilinx [31] is employed as a software tool for the design of the presented MPA coprocessor. In the design process, simulation waveforms are obtained with the use of Aldec Riviera tool [34]. The MPA coprocessor is implemented in Xilinx Zynq-7000 SoC on TySOM-1 board from Aldec [27]. The benchmarking setup for the MPA coprocessor is presented in Figure 12, whereas the picture of the benchmarking hardware is presented in Figure 13. The benchmarking codes can be executed either on CPU or FPGA. Then, the external host computer is used to manage benchmarks and acquire execution times. The reference CPU codes are executed on ARM Cortex A9 on TySOM-1 board, which operates under the control of Linux RTOS (i.e., PetaLinux) loaded from an SD card. Runtimes are measured on CPU using the system clock. In order to measure runtimes on FPGA, the external host computer communicates with the integrated logic analyser (ILA) [35] within FPGA using JTAG interface. ILA is implemented within FPGA as an IP core and is used to monitor internal signals and registers of a design. As mentioned, the time of the code execution is measured on the MPA coprocessor with the use of timer. This timer is triggered by the rising edge of the clock at the beginning of the execution of the first code instruction whilst the end of computations is triggered by the first transfer of resulting data. A value in the timer is acquired using ILA and transferred then to the host computer. The entire process of receiving results is handled from the level of Xilinx Vivado tool (i.e., Program \& Debug tab). The results are downloaded using the JTAG server working as "daemon tool" and placed on time charts of the signal state analyser. The results presented in Figures 9-11 are taken for the values of the parameter $n$, which allow us to process the measurement results within a reasonable time.

The results for ARM Cortex A9 are obtained using CPU codes compiled with the use of the GNU CC compiler (version 4.9.2, disabled all optimizations). The following compilation options were additionally considered for the code optimization on CPU: -O2, -O3, -Ofast, -Os, -Og. These options allow us to speed up the code execution, reduce the code size as well as support the code debugging. In comparison to the compilations with disabled all optimizations, runtimes are maximally reduced by $5 \%$ for computations of factorials and $n$-th power of $n(n \geq 200)$, whereas runtimes are maximally reduced by $3 \%$ for DGF computations $(n \geq 300)$. Therefore, the results are presented for the CPU codes compiled with all optimizations disabled.

The design flow of the developed MPA coprocessor is presented in Figure 14. Based on the design requirements, the development of the coprocessor architecture and test environment start simultaneously. Afterwards, basic blocks (i.e, multiplier, adder-subtractor, registers, etc.) are developed which are then integrated into the coprocessor. The developed coprocessor codes are tested in behavioural simulations (using VHDL codes) and functional simulations (using SystemVerilog (SV) and $C$ codes). Afterwards, the coprocessor is benchmarked in simulations and its performance is compared to the ARM Cortex A9 core with the GMP library. The feedback from the design process allows us to modify the design to obtain the design requirements. Finally, the MPA coprocessor is verified and benchmarked on hardware. 


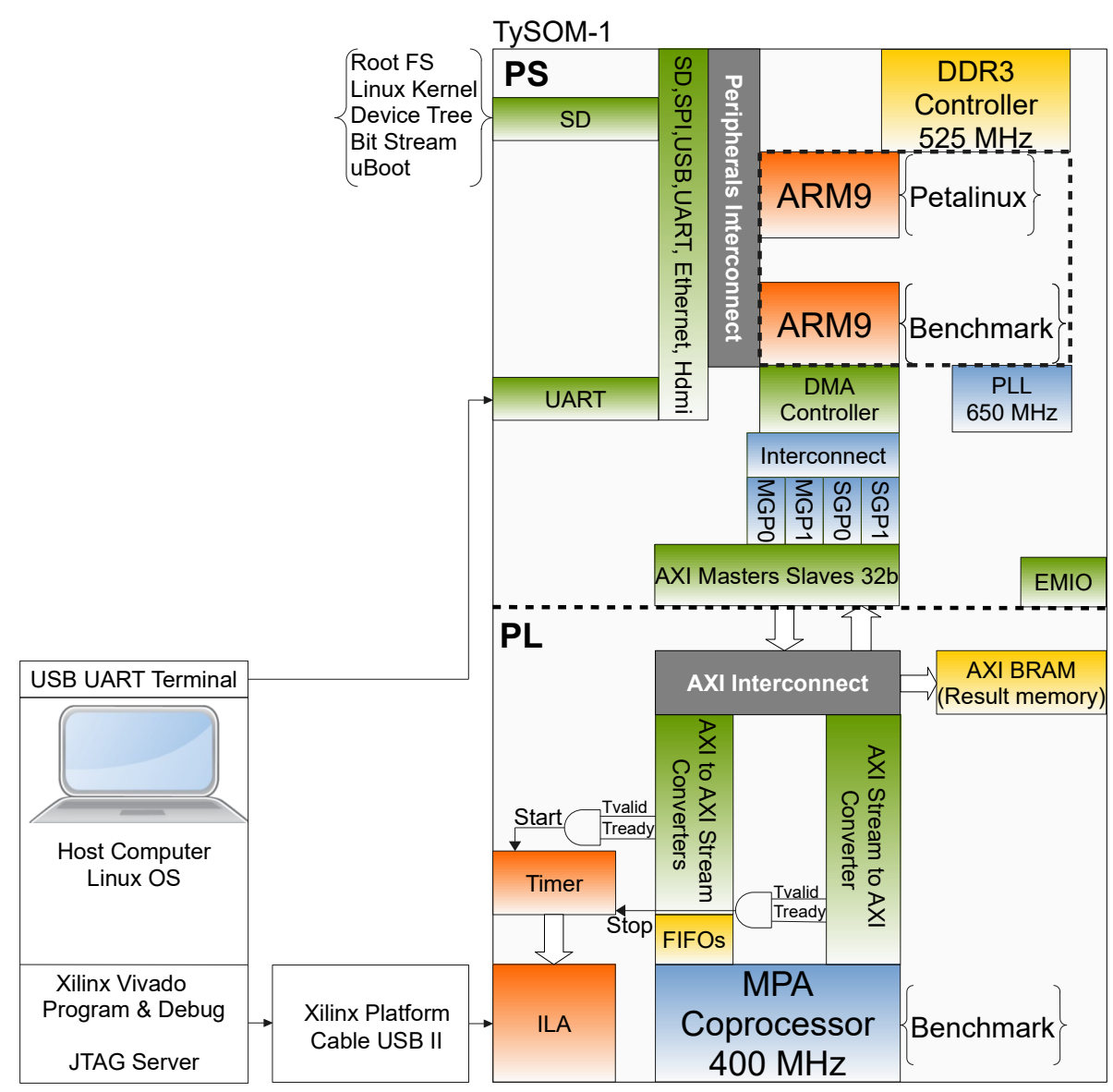

Figure 12. Benchmarking setup for MPA coprocessor.

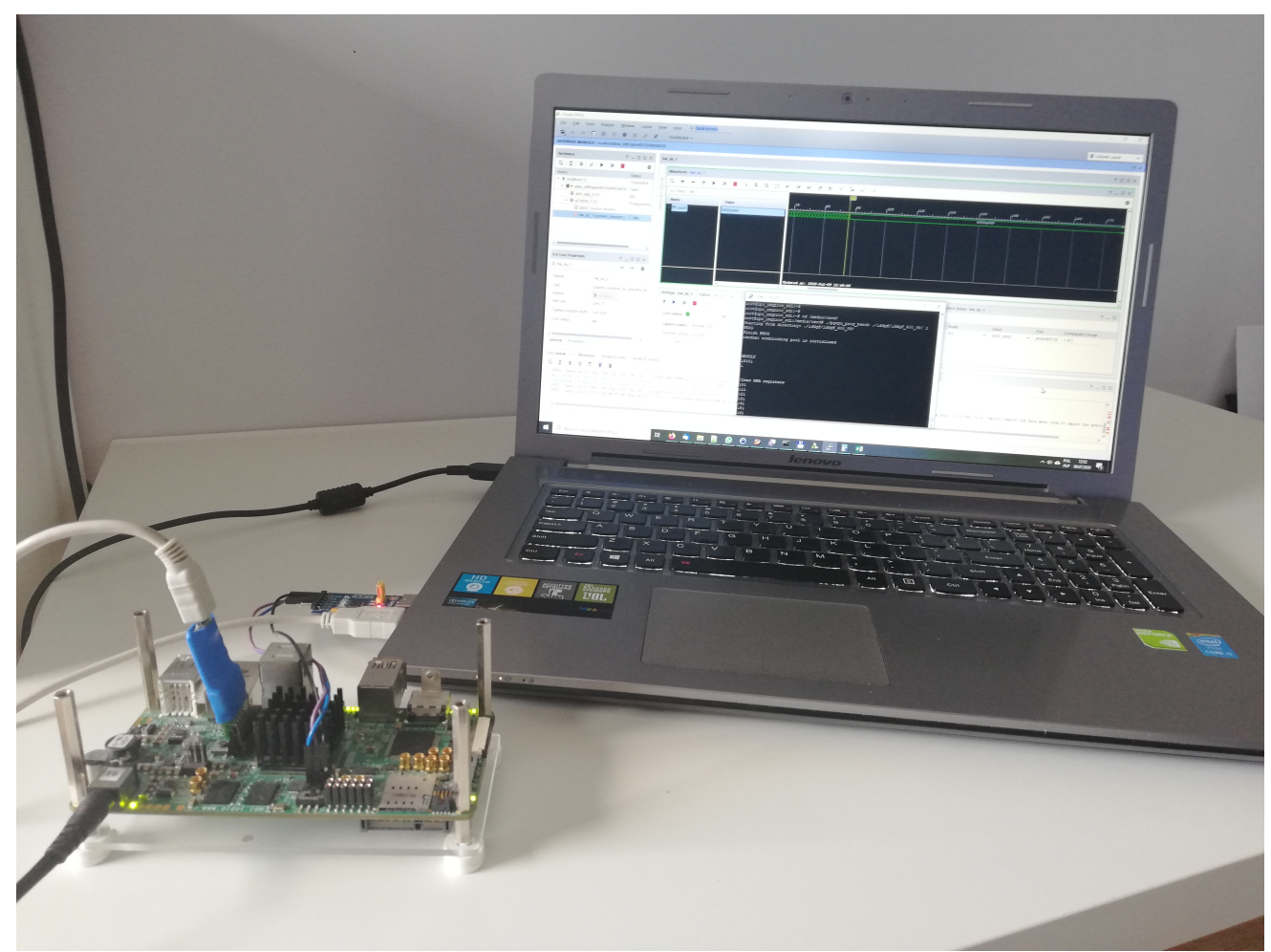

Figure 13. Picture of benchmarking hardware for MPA coprocessor. 


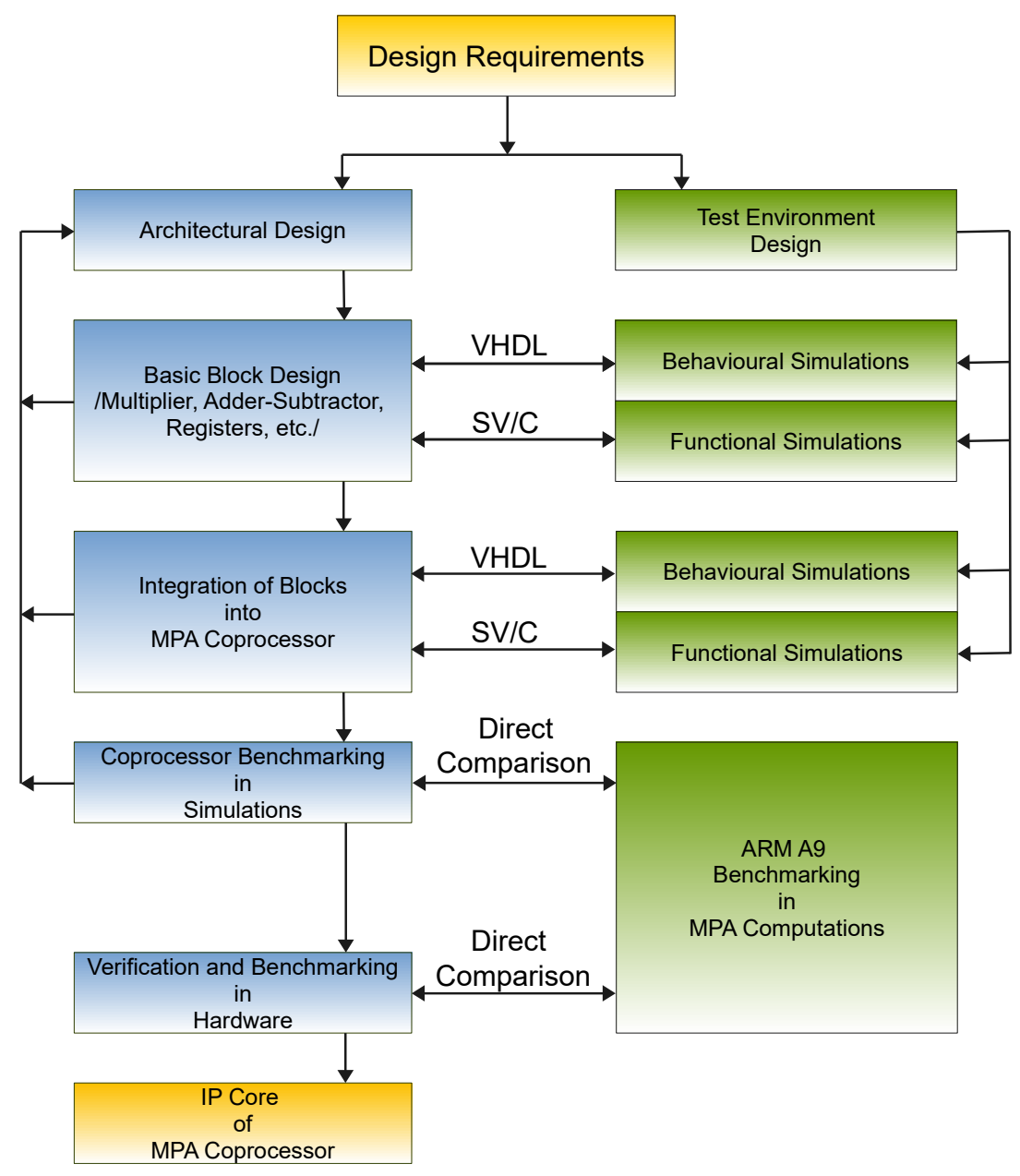

Figure 14. Design flow of MPA coprocessor.

\section{Conclusions}

Our open-source coprocessor is intended to support MPA computations by offloading them from the CPU, with precision varying in the range between 64 bits and $32 \mathrm{kbits}$, with the limb size set to 64 bits. In our solution, MPA numbers are stored with the use of the sign-magnitude representation to increase efficiency of the multiplication operation as well as to enable compatibility with existing software MPA libraries. The developed coprocessor is benchmarked in factorial, exponentiation and DGF computations, using an FPGA implementation on Xilinx Zynq-7000 SoC on TySOM-1 board from Aldec. In the factorial and exponentiation benchmarks, our coprocessor can be three times faster than a single CPU core within the same chip for sufficiently large input parameters. In the task of DGF generation, the MPA coprocessor is more than ten times faster than a single CPU core within the same chip for sufficiently large input parameters. With no more than $80 \%$ resource utilization, one can implement six MPA cores on Zynq-7000 SoC. The speedup is obtained as a result of aggressive optimization of performance and resource consumption. It means that over $87 \%$ of the maximum operating frequency is obtained for the bottleneck element in FPGA (BRAM), whose maximum operating frequency is equal to $458 \mathrm{MHz}$. It is clearly demonstrated that the embedding of MPA computations in programmable logic within $\mathrm{SoC}$ is an attractive direction for the future development of MPA computations accelerator. Our MPA coprocessor can facilitate other researchers involved in MPA scientific computing, because the VHDL code of the MPA coprocessor is open-sourced, along with all emulation and benchmarking codes presented in this paper. The next steps of this work are to 
develop a bit manipulation unit, apply the coprocessor for data encryption, and integrate it with the RISC-V processor.

Author Contributions: Conceptualization, K.R. and T.P.S.; methodology, K.R. and T.P.S.; firmware and software, K.R. and T.P.S.; validation, K.R., T.P.S. and W.Ż.; investigation, K.R., T.P.S. and W.Ż.; data curation, W.Ż.; writing—original draft preparation, K.R., T.P.S. and W.Ż.; writing—review and editing, K.R., T.P.S. and W.Ż. All authors have read and agreed to the published version of the manuscript.

Funding: This research received no external funding.

Acknowledgments: Tomasz Stefański is grateful to Cathal McCabe at Xilinx Inc. for arranging the donation of design software tools.

Conflicts of Interest: The authors declare no conflict of interest.

\section{References}

1. Bailey, D.; Barrio, R.; Borwein, J. High-precision computation: Mathematical physics and dynamics. Appl. Math. Comput. 2012, 218, 10106-10121. [CrossRef]

2. Stefański, T.P. Electromagnetic Problems Requiring High-Precision Computations. IEEE Antennas Propag. Mag. 2013, 55, 344-353. [CrossRef]

3. Stefanski, T.P. Fast Implementation of FDTD-Compatible Green's Function on Multicore Processor. IEEE Antennas Wirel. Propag. Lett. 2012, 11, 81-84. [CrossRef]

4. Stefański, T.P.; Krzyzanowska, K. Implementation of FDTD-Compatible Green's Function on Graphics Processing Unit. IEEE Antennas Wirel. Propag. Lett. 2012, 11, 1422-1425. [CrossRef]

5. Stefanski, T.P. Implementation of FDTD-Compatible Green's Function on Heterogeneous Cpu-GPU Parallel Processing System. Prog. Electromagn. Res. 2013, 135, 297-316. [CrossRef]

6. Ergül, Ö.; 1 şcan Karaosmanoğlu, B. Low-Frequency Fast Multipole Method Based on Multiple-Precision Arithmetic. IEEE Antennas Wirel. Propag. Lett. 2014, 13, 975-978. [CrossRef]

7. Kalfa, M.; Ergül, Ö; Ertürk, V.B. Error Control of Multiple-Precision MLFMA. IEEE Trans. Antennas Propag. 2018, 66, 5651-5656. [CrossRef]

8. Kalfa, M.; Ertürk, V.B.; Ergül, Ö. Error Control of MLFMA within a Multiple- Precision Arithmetic Framework. In Proceedings of the 2018 IEEE International Symposium on Antennas and Propagation USNC/URSI National Radio Science Meeting, Boston, MA, USA, 8-13 July 2018; pp. 2299-2300.

9. Wolfe, J.M. Reducing Truncation Errors by Programming. Commun. ACM 1964, 7, 355-356. [CrossRef]

10. Kahan, W. Pracniques: Further Remarks on Reducing Truncation Errors. Commun. ACM 1965, 8, 40 [CrossRef]

11. Goodman, J.; Heggie, D.C.; Hut, P. On the Exponential Instability of N-Body Systems. Astrophys. J. 1993, 415, 715. [CrossRef]

12. Gustafson, J. The End of Error: Unum Computing; CRC Press: Boca Raton, FL, USA, 2017.

13. Tenca, A.F.; Ercegovac, M.D. A variable long-precision arithmetic unit design for reconfigurable coprocessor architectures. In Proceedings of the IEEE Symposium on FPGAs for Custom Computing Machines (Cat. No.98TB100251), Napa Valley, CA, USA, 17 April 1998; pp. 216-225. [CrossRef]

14. Schulte, M.J.; Swartzlander, E.E. A family of variable-precision interval arithmetic processors. IEEE Trans. Comput. 2000, 49, 387-397. [CrossRef]

15. Lei, Y.; Dou, Y.; Guo, S.; Zhou, J. FPGA Implementation of Variable-Precision Floating-Point Arithmetic. In Advanced Parallel Processing Technologies; Temam, O., Yew, P.C., Zang, B., Eds.; Springer: Berlin/Heidelberg, Germany, 2011; pp. 127-141.

16. Daisaka, H.; Nakasato, N.; Makino, J.; Yuasa, F.; Ishikawa, T. GRAPE-MP: An SIMD Accelerator Board for Multi-precision Arithmetic. Procedia Comput. Sci. 2011, 4, 878-887. [CrossRef]

17. Daisaka, H.; Nakasato, N.; Ishikawa, T.; Yuasa, F. Application of GRAPE9-MPX for High Precision Calculation in Particle Physics and Performance Results. Procedia Comput. Sci. 2015, 51, 1323-1332. [CrossRef]

18. Bocco, A.; Durand, Y.; De Dinechin, F. SMURF: Scalar Multiple-Precision Unum Risc-V Floating-Point Accelerator for Scientific Computing. In CoNGA'19, Proceedings of the Conference for Next Generation Arithmetic 2019; Association for Computing Machinery: New York, NY, USA, 2019. [CrossRef] 
19. Bocco, A.; Durand, Y.; de Dinechin, F. Dynamic Precision Numerics Using a Variable-Precision UNUM Type I HW Coprocessor. In Proceedings of the 2019 IEEE 26th Symposium on Computer Arithmetic (ARITH), Kyoto, Japan, 10-12 June 2019; pp. 104-107.

20. Bocco, A.; Jost, T.T.; Cohen, A.; de Dinechin, F.; Durand, Y.; Fabre, C. Byte-Aware Floating-point Operations through a UNUM Computing Unit. In Proceedings of the 2019 IFIP/IEEE 27th International Conference on Very Large Scale Integration (VLSI-SoC), Cuzco, Peru, 6-9 October 2019; pp. 323-328.

21. Asanović, K.; Avizienis, R.; Bachrach, J.; Beamer, S.; Biancolin, D.; Celio, C.; Cook, H.; Dabbelt, D.; Hauser, J.; Izraelevitz, A.; et al. The Rocket Chip Generator; Technical Report UCB/EECS-2016-17; EECS Department, University of California: Berkeley, CA, USA, 2016.

22. Rudnicki, K.; Stefański, T.P. FPGA implementation of the multiplication operation in multiple-precision arithmetic. In Proceedings of the 2017 MIXDES-24th International Conference Mixed Design of Integrated Circuits and Systems, Bydgoszcz, Poland, 22-24 June 2017; pp. 271-275. [CrossRef]

23. Rudnicki, K.; Stefanski, T.P. IP Core of Coprocessor for Multiple-Precision-Arithmetic Computations. In Proceedings of the 2018 25th International Conference Mixed Design of Integrated Circuits and System (MIXDES), Gdynia, Poland, 21-23 June 2018; pp. 416-419. [CrossRef]

24. Rudnicki, K.; Stefanski, T.P. Implementation of Addition and Subtraction Operations in Multiple Precision Arithmetic. In Proceedings of the 2019 26th International Conference Mixed Design of Integrated Circuits and System (MIXDES), Rzeszów, Poland, 27-29 June 2019.

25. Xilinx Inc. Zynq-7000 All Programmable SoC Data Sheet: Overview_Product Specification. 2017. Available online: www.xilinx.com (accessed on 8 August 2019).

26. Rudnicki, K.; Stefański, T.P.; Żebrowski, W. Integer-MPA-Coprocessor. 2020. Available online: https: / / github.com/stafan26/integer-MPA-coprocessor (accessed on 13 July 2020).

27. Aldec Inc. TySOM-1-7Z030-Technical Specification, Revision 1.7. 2017. Available online: www.aldec.com (accessed on 8 August 2019).

28. Xilinx Inc. Vivado Design Suite-AXI Reference Guide, UG1037 (v4.0). 2017. Available online: www.xilinx. com (accessed on 8 August 2019).

29. Granlund, T.; GMP Development Team. The GNU Multiple Precision Arithmetic Library (Edition 6.1.2). 2016. Available online: www.gmplib.org (accessed on 8 August 2019).

30. Xilinx Inc. 7 Series DSP48E1 Slice-User Guide, UG479 (v1.10). 2018. Available online: www.xilinx.com (accessed on 8 August 2019).

31. Xilinx Inc. Vivado Design Suite User Guide-Getting Started, UG910 (v2018.3). 2018. Available online: www.xilinx.com (accessed on 8 August 2019).

32. Gulgowski, J.; Stefański, T.P. Recurrence scheme for FDTD-compatible discrete Green's function derived based on properties of Gauss hypergeometric function. J. Electromagn. Waves Appl. 2019, 33, 637-653. [CrossRef]

33. Taflove, A.; Hagness, S.C. Computational Electrodynamics: The Finite-Difference Time-Domain Method, 3rd ed.; Artech House: Norwood, MA, USA, 2005.

34. Aldec Inc. Riviera-PRO Manual. 2017. Available online: www.aldec.com (accessed on 8 August 2019).

35. Xilinx Inc. Integrated Logic Analyzer v6.2-LogiCORE IP Product Guide, PG172. 2016. Available online: www.xilinx.com (accessed on 8 August 2019).

(C) 2020 by the authors. Licensee MDPI, Basel, Switzerland. This article is an open access article distributed under the terms and conditions of the Creative Commons Attribution (CC BY) license (http://creativecommons.org/licenses/by/4.0/). 